TRANSACTIONS OF THE

AMERICAN MATHEMATICAL SOCIETY

Volume 350, Number 4, April 1998, Pages 1429-1466

S 0002-9947(98)02052-2

\title{
FOX CALCULUS, SYMPLECTIC FORMS, AND MODULI SPACES
}

\author{
VALENTINO ZOCCA
}

\begin{abstract}
An "open pre-symplectic form" on surfaces with boundary and glueing formulae are provided to symplectically integrate the symplectic form on the deformation space of representations of the fundamental group of a Riemann surface into a reductive Lie group $G$.
\end{abstract}

Moduli spaces of flat connections over a Riemann surface, or their equivalent interpretation as moduli spaces of faithful, irreducible representations of the fundamental group of a Riemann surface, have been extensively studied. These spaces can also be referred to as spaces of $G$-structures, since they depend on a group $G$ : in the interpretation as moduli spaces of flat connections on a bundle $E, G$ represents the structural group of the bundle; in the interpretation as moduli spaces of faithful, irreducible representations, the group $G$ represents the codomain for the representation.

These spaces carry a natural symplectic structure which, for $G=\operatorname{PSL}(2, \mathbf{R})$, was studied and symplectically integrated by Scott Wolpert ([Wol85]). That means that he explicitly expressed such symplectic form in terms of global coordinates, the Fenchel-Nielsen coordinates, defined on the moduli space of $\operatorname{PSL}(2, \mathbf{R})$-structures, also known as Teichmüller space.

The theory on the symplectic structure on moduli spaces of $G$-structures has been widely developed by many authors. Most of this theory has been approached using a complex-differential-geometric, or an algebraic-geometric, point of view.

It is the project of the author to show, in this article, another possible approach to the study of the symplectic structure on moduli spaces of $G$-structures.

This approach, to the author's knowledge, was initiated by William Goldman ([Gol84]). To a Riemann surface we associate a generator in $H_{2}(\Sigma)$, the fundamental class of $\Sigma$. The tangent space of the moduli space of representations $\operatorname{Hom}\left(\pi_{1}(\Sigma), G\right) / G$ is denoted by $H^{1}\left(\pi_{1}(\Sigma), \mathcal{G}_{A d_{\phi}}\right)$ and represents the first groupcohomology space with coefficients in the Lie algebra $\mathcal{G}$ of $G$.

The object of this paper is then to show how it is possible to completely symplectically integrate the symplectic form $\omega$ on these moduli spaces in terms of a set of global coordinates defined on them. In particular, the cases $G=\operatorname{PSL}(2, \mathbf{R})$ and $G=\operatorname{PGL}(3, \mathbf{R})$ will be explicitly treated as examples.

This article represents a step forward towards a better understanding of the symplectic structure of those spaces. For compact Riemann surfaces, both spaces, as in general any space of irreducible representations $\operatorname{Hom}\left(\pi_{1}(\Sigma), G\right) / G$, with $G$

Received by the editors May 15, 1996.

1991 Mathematics Subject Classification. Primary 58D27.

Supported by a C.N.R. grant. 
any semisimple Lie group, are symplectic manifolds of dimension $2(g-1) \operatorname{dim}(G)$. These spaces have an equivalent interpretation via holonomy, as moduli spaces of flat irreducible connections over a $G$-bundle on the Riemann surface $\Sigma$ ([GM88]).

Using this second interpretation, its tangent space is represented by the first group-cohomology group $H^{1}\left(\pi_{1}(\Sigma), \mathcal{G}_{A d_{\phi}}\right)$.

The symplectic form $\omega$, defined on the moduli space of flat connections, will then act by

$$
\omega(\alpha, \beta)=-\int_{\Sigma} K(\alpha \wedge \beta),
$$

where $\alpha, \beta \in H^{1}(\Sigma, \mathcal{G})$ and $K$ represents the Killing form.

For most of our examples, concerning groups of matrices, the Killing form will just be the trace.

N.B. More generally, we can assume that $G$ is reductive and $K$ is the nondegenerate, symmetric, bilinear form defined on its Lie-algebra.

Analogously, the symplectic form $\omega$ can be described by the formula

$$
\omega(u, v)=\sum_{j} K\left(u\left(\# \frac{\partial R}{\partial x_{j}}\right), v\left(x_{j}\right)\right),
$$

where $K$ represents the Killing formula on the Lie algebra $\mathcal{G}$ of $G, u$ and $v$ are elements in the tangent space of $\operatorname{Hom}\left(\pi_{1}(\Sigma), G\right) / G$, and $\sum_{j}\left(\frac{\partial R}{\partial x_{j}}, x_{j}\right) \in H_{2}\left(\pi_{1}(\Sigma)\right)$ describes the fundamental 2-chain representing $\Sigma$. For $\operatorname{PSL}(2, \mathbf{R})$ and $\operatorname{PGL}(3, \mathbf{R})$ we have that $K=$ trace.

All this is discussed in chapter 1.

The formula for $\omega$ and the expression for the fundamental 2-chain of $\Sigma$ can be extended to open Riemann surfaces. However $\omega$ will not define a symplectic form anymore but just a bilinear form that we will call the open pre-symplectic form.

The tangent space to $\operatorname{Hom}\left(\pi_{1}(\Sigma), G\right) / G$ at a representation $\phi$ is the first groupcohomology space $H^{1}\left(\pi_{1}(\Sigma), \mathcal{G}_{A d \phi}\right)$. The space $H^{1}\left(\pi_{1}(\Sigma), \mathcal{G}_{A d \phi}\right)$ is a space of lateral classes of 1-cocycles. A 1-cocycle $u$ is defined to be a map $u: \pi_{1}(\Sigma) \rightarrow \mathcal{G}$ satisfying the relation $u(\gamma \eta)=u(\gamma)+\phi(\gamma) u(\eta) \phi(\gamma)^{-1} ; \gamma, \eta \in \pi_{1}(\Sigma)$. For compact Riemann surfaces the definition of $\omega$ is independent of the choice of the representant $u$ for each class $[u] \in H^{1}\left(\pi_{1}(\Sigma), \mathcal{G}_{A d \phi}\right)$; however, the open pre-symplectic form does depend on this choice and is therefore only defined on the space of 1 -cocycles: $C^{1}\left(\pi_{1}(\Sigma), \mathcal{G}_{\text {Ad }}\right)$.

The dependence of $\omega$ on the choice of the representant is determined by the formula

$$
\omega\left(d u_{0}, v\right)=\operatorname{tr}\left(u_{0}, v(\delta \Sigma)\right),
$$

where $d u_{0}$ is an exact 1-cocycle and $\delta \Sigma=$ boundary of $\Sigma$. Also, the open presymplectic form $\omega$ is not skew-symmetric, and this is expressed by the formula

$$
\omega(u, v)+\omega(v, u)=\sum \operatorname{tr}\left(u\left(c_{i}\right), v\left(c_{i}\right)\right),
$$

where $\sum c_{i}=\delta \Sigma$.

The advantage of defining the fundamental 2-chain and the open pre-symplectic form for open Riemann surfaces is that we can study them on open surfaces and then study how they behave under glueing. Glueing formulae for connected sum glueings (or group amalgamation) and HNN-glueings are provided.

Given two Riemann surfaces $\Sigma^{\prime}\left(g^{\prime}, n^{\prime}\right)$ and $\Sigma^{\prime \prime}\left(g^{\prime \prime}, n^{\prime \prime}\right)$ and their connected sum $\Sigma(g+g, n+n-2)$, we find the following formula for combining the fundamental 
classes:

$$
F C(\Sigma)=F C\left(\Sigma^{\prime}\right)+\Phi_{R_{1}}\left(F C\left(\Sigma^{\prime \prime}\right)\right)-\left(R_{1}, c_{1}\right)-\left(R_{1} R_{2}, c_{2}\right)
$$

as well as the formula for combining the open pre-symplectic form:

$$
\omega(u, v)=\omega^{\prime}(u, v)+\omega^{\prime \prime}(u, v)-\operatorname{trace}\left(u\left(c^{\prime}\right) \cdot v\left(c^{\prime \prime-1}\right)\right),
$$

where $F C$ is the fundamental class, $\omega$ the open pre-symplectic form, $R_{1} c_{1}=$ id and $R_{2} c_{2}=$ id represent the relations for the fundamental group of $\Sigma^{\prime}$ and $\Sigma^{\prime \prime}$ respectively, $c^{\prime}$ and $c^{\prime \prime}$ are the boundary components of $\Sigma^{\prime}$ and $\Sigma^{\prime \prime}$ respectively, and $\Phi_{R_{1}}$ is a chain operator.

The chain operator is defined on fundamental 2-chains by:

$$
\Phi_{R}\left(\sum\left(A_{i}, B_{i}\right)\right)=\sum\left(R A_{i}, B_{i}\right) .
$$

If we let $\Sigma$ be a Riemann surface and $\hat{\Sigma}$ be the resulting Riemann surface after having glued two boundary components of $\Sigma$, for an HNN-glueing the associated formulae are

$$
F C(\hat{\Sigma})=F C(\Sigma)-\left(R c_{1}, c_{2}\right)+\left(R c_{1}, x\right)-(1, x)-\left(x, c_{1}\right)
$$

and

$$
\begin{aligned}
& \hat{\omega}(u, v)=\omega(u, v)-\operatorname{tr}\left(u\left(\# R c_{1}\right) \cdot v\left(\# R c_{1}\right)\right) \\
& +\operatorname{tr}\left(u\left(\# R c_{1}\right) \cdot v(x)\right)-\operatorname{tr}\left(u(\# x) \cdot v\left(c_{1}\right)\right)
\end{aligned}
$$

where again $F C$ represents the fundamental class, $\omega$ the open pre-symplectic form on $\Sigma, \hat{\omega}$ the open pre-symplectic form on $\hat{\Sigma}, R c_{1} c_{2}=\mathrm{id}$, and $R\left[c_{1}, x\right]=$ id represent the relations for the fundamental group of $\Sigma$ and $\hat{\Sigma}$ respectively, $c_{1}$ and $c_{2}$ are the boundary components of $\Sigma$, and $x$ the loop on $\hat{\Sigma}$ generated by the HNN-glueing. The open pre-symplectic form and its glueing formulae are discussed in chapter 2 .

We then focus on the explicit calculation of the open pre-symplectic form. Chapters 3 and 4 are devoted to its study on open Riemann surfaces, and discuss the use of the glueing formulae to explicitly integrate the symplectic form. The cases $G=\operatorname{PSL}(2, \mathbf{R})$ and $G=\operatorname{PGL}(3, \mathbf{R})$ are considered.

For $G=\operatorname{PSL}(2, \mathbf{R})$ we use the Fenchel-Nielsen coordinates defined on Teichmüller space. By explicitly calculating the 1-cocycles relative to the tangent vectors defined by differentiation along the directions relative to such coordinates, we find an explicit expression for the open pre-symplectic form in the $\operatorname{PSL}(2, \mathbf{R})$ case. This reproves and extends results by Wolpert on the symplectic form on Teichmüller space, which he proved to be $\omega=\sum\left(d l_{i} \wedge d \theta_{i}\right)$, with $l_{i}$ and $\theta_{i}$ the Fenchel-Nielsen coordinates. Also, an explicit application of the glueing formula is given. This is discussed in chapter 3 .

Then we consider the PGL $(3, \mathbf{R})$ case. In particular we need to define new coordinates that extend the Fenchel-Nielsen coordinates to the deformation space of convex real projective structures, and we use results by Goldman. The PGL $(3, \mathbf{R})$ case is referred to as the deformation space of convex real projective structures. The deformation space of convex real projective structures on a Riemann surface $\Sigma$ represents a generalisation of the deformation space of hyperbolic structures, Teichmüller space. While Teichmüller space is described by the space of irreducible, faithful representations from $\pi_{1}(\Sigma)$ to $\operatorname{PSL}(2, \mathbf{R})$, modulo conjugation, i.e. an open subset of $\operatorname{Hom}\left(\pi_{1}(\Sigma), \operatorname{PSL}(2, \mathbf{R})\right) / \operatorname{PSL}(2, \mathbf{R})$, the space of convex real 
projective structures is an open subset of $\operatorname{Hom}\left(\pi_{1}(\Sigma), \operatorname{PGL}(3, \mathbf{R})\right) / \operatorname{PGL}(3, \mathbf{R})$ and there exists an inclusion of the former into the latter.

The definitions of and the relations between the two spaces are described in the first part of chapter 4 . In the remainder of chapter 4 we turn to the explicit calculation of the open pre-symplectic form on the deformation space of convex real projective structures on a trinion. As in chapter 3, this is done by explicitly calculating, for these new coordinates, the corresponding 1-cocycles, and by evaluating the formula for the open pre-symplectic form on them.

This represents the first explicit calculation and expression of the open presymplectic form on the deformation space of projective structures. This process can also be suitably extended to calculate the symplectic form on the moduli spaces of representations $\operatorname{Hom}\left(\pi_{1}(\Sigma), G\right) / G$, with $G$ representing any classical semisimple group of matrices, e.g. $\mathrm{SU}(n), \mathrm{U}(n)$, et cetera.

\section{ACKNOWLEGEMENTS}

Much of the work contained in this paper has been inspired by fertile and mathematically advantageous discussions with Dr. Scott Wolpert. My work has also been enriched by interesting discussions with Dr. William Goldman and Dr. John Millson. In particular Dr. Goldman explained to me much of his earlier results, and his help was invaluable.

I would also like to thank the Mathematics Department of the University of Maryland for offering me a productive environment to realise this work. The Department of Mathematics at the University of Paris/Orsay, France, provided me with a welcoming atmosphere for the final write-up.

\section{The moduli space And its tangent SPACE}

Let $\Sigma$ be a closed oriented topological surface of genus $g \geq 1$, let $\pi_{1}(\Sigma)$ be its fundamental group, and let $G$ be a reductive Lie group, i.e. a Lie group that admits a non-degenerate bilinear form on it, or the Killing form for semi-simple Lie groups.

Let $\operatorname{Hom}\left(\pi_{1}(\Sigma), G\right) / G$ denote the space of representations (or homomorphisms) with the compact-open topology.

The finite group $\pi_{1}(\Sigma)$ can be expressed as the quotient $\Pi /\langle R\rangle$, where $\Pi$ is the free group with $2 g$ generators $\left\{a_{1}, \cdots, a_{g}, b_{1}, \cdots, b_{g}\right\}$ and $\langle R\rangle$ represents the free cyclic normal subgroup generated by the element $R=\left[a_{1}, b_{1}\right] \cdot\left[a_{2}, b_{2}\right] \cdots\left[a_{g}, b_{g}\right]$, where $\left[a_{j}, b_{j}\right]=a_{j} b_{j} a_{j}^{-1} b_{j}^{-1}$ is the commutator of $a_{j}$ and $b_{j}$ as usual.

The group $G$ acts on $\operatorname{Hom}\left(\pi_{1}(\Sigma), G\right)$ by inner automorphisms (conjugation), the action being

$$
g: \phi \mapsto \phi^{g}
$$

where $\phi^{g}: \gamma \mapsto g \cdot \phi(\gamma) \cdot g^{-1}$.

We restrict our attention to the space of representations $\phi$ such that the centraliser of $\phi\left(\pi_{1}(\Sigma)\right)$ in $G$, modulo the center of $G$, is zero-dimensional.

Theorem 1.0.1 ([Gol84]). Let $G$ be a semi-simple Lie group. The set of all representations $\phi \in \operatorname{Hom}\left(\pi_{1}(\Sigma), G\right) / G$ such that $\operatorname{dim}\left[Z\left(\phi\left(\pi_{1}(\Sigma)\right)\right) / Z(G)\right]=0$ represents the set of simple points $\operatorname{Hom}^{s}\left(\pi_{1}(\Sigma), G\right)$, whose dimension equals

$$
(2 \cdot \operatorname{genus}(\Sigma)-1) \cdot \operatorname{dim}(G) .
$$


The condition that $\operatorname{dim}[Z(\phi) / Z(G)]=0$ is equivalent, for $G=\mathrm{U}(n)$, to the condition that $\phi$ be irreducible and in general to the stable points as discussed in Mumford's Geometric Invariant Theory. If $G$ is compact, then $\operatorname{Hom}^{s}\left(\pi_{1}(\Sigma), G\right) / G$ represents a Hausdorff space, and then a manifold; however, if $G$ is not compact, $\operatorname{Hom}^{s}\left(\pi_{1}(\Sigma), G\right) / G$ need not be Hausdorff.

In the non-compact case we need to restrict ourselves to the representations $\phi$ on which $G / Z(G)$ acts properly (i.e. the orbits are separated). Then $\operatorname{Hom}^{s}\left(\pi_{1}(\Sigma), G\right) / G$ is a Hausdorff space of dimension $(2 \cdot \operatorname{genus}(\Sigma)-2) \cdot \operatorname{dim}(G)+$ $2 \cdot \operatorname{dim}(Z(G))([\mathrm{JM} 84])$.

Since $\operatorname{Hom}^{s}\left(\pi_{1}(\Sigma), G\right) / G$ is not Hausdorff in general, we need to replace it with its maximal Hausdorff subspace by identifying points that cannot be separated. Henceforth whenever we write $\operatorname{Hom}\left(\pi_{1}(\Sigma), G\right) / G$ we will refer to this manifold.

The space $\operatorname{Hom}\left(\pi_{1}(\Sigma), G\right) / G$ is equivalent to the moduli space of flat connections on a $G$-bundle ([GM88]). If we use the flat connection interpretation, then its tangent space at a point (or connection) $A$ corresponds to the first cohomology group $\mathrm{H}_{A}^{1}(\Sigma, \mathcal{G})$.

In fact if $\mathcal{A}=\{$ space of flat connections on a $G$-bundle $E$ over $\Sigma\}, \mathcal{A}$ is an affine space whose tangent space is given by the space of connection 1-forms $\alpha \in C^{1}(\Sigma, \mathcal{G})$; since we are considering flat connections we have the extra condition $d_{A} \alpha=0$, i.e. $\alpha$ is also closed, i.e. it descends to an element in $H_{A}^{1}(\Sigma, \mathcal{G})$.

Following the algebraic interpretation of these spaces, we can describe the tangent space of $\operatorname{Hom}\left(\pi_{1}(\Sigma), G\right) / G$ as the cohomology space $H^{1}\left(\pi_{1}(\Sigma), \mathcal{G}_{A d_{\phi}}\right)$.

Theorem 1.0.2. The tangent space at an equivalence class of representations $[\phi]$ to $\operatorname{Hom}\left(\pi_{1}(\Sigma), G\right) / G$ is given by $H^{1}\left(\pi_{1}(\Sigma), \mathcal{G}_{A d_{\phi}}\right)$.

Proof. Given a representation $\phi \in \operatorname{Hom}\left(\pi_{1}(\Sigma), G\right)$, its first order approximation is given by $\phi_{t}(x)=\exp \left(t u(x)+O\left(t^{2}\right)\right) \phi(x)$, where $u: \pi_{1}(\Sigma) \rightarrow \mathcal{G}_{A d_{\phi}}$ satisfies the cocycle condition $u(x y)=u(x)+A d_{\phi(x)} u(y)$ (see [Gol84] for more details). The space of 1-cocycles $u, Z^{1}\left(\pi_{1}(\Sigma), \mathcal{G}_{A d_{\phi}}\right)$, then represents the tangent space to $\operatorname{Hom}\left(\pi_{1}(\Sigma), G\right)$.

Since we are interested in the deformation space $\operatorname{Hom}\left(\pi_{1}(\Sigma), G\right) / G$ we now turn to the action of $G$ on $\operatorname{Hom}\left(\pi_{1}(\Sigma), G\right)$ by inner automorphisms. A trivial deformation on $\operatorname{Hom}\left(\pi_{1}(\Sigma), G\right) / G$ is a deformation $\phi_{t}(x)$ induced by a path $g_{t}$ in $G$, i.e. $\phi_{t}(x)$ $=g_{t} \phi(x) g_{t}^{-1}$. The first order approximation $g_{t}=\exp \left(t u_{0}+O\left(t^{2}\right)\right)$ implies

$$
\begin{aligned}
\phi_{t}(x) & =\exp \left(t u_{0}+O\left(t^{2}\right)\right) \phi(x) \exp \left(-t u_{0}-O\left(t^{2}\right)\right) \\
& =\exp \left(t u_{0}+O\left(t^{2}\right)\right) \phi(x) \exp \left(-t u_{0}-O\left(t^{2}\right)\right) \phi(x)^{-1} \phi(x) \\
& =\exp \left(t\left(u_{0}-A d_{\phi(x)} u_{0}\right)+O\left(t^{2}\right)\right) \phi(x) .
\end{aligned}
$$

Therefore a trivial deformation $\phi_{t}$ is given by a 1-cocycle $u=u_{0}-A d_{\phi(x)} u_{0}$ which represents the coboundary $\delta u_{0}$ in group cohomology of a constant 0 -cycle $u_{0}$ : $\pi_{1}(\Sigma) \rightarrow \mathcal{G}$. Hence $H^{1}\left(\pi_{1}(\Sigma), \mathcal{G}_{A d_{\phi}}\right)=$ (1-cocycles)/(exact 1-cocycles) represents the tangent space of $\operatorname{Hom}\left(\pi_{1}(\Sigma), G\right) / G$ (see [Gol84]).

The tangent space to the Hausdorff manifold of flat connections, modulo gauge transformations, is given by the first cohomology vector space $H_{A}^{1}(\Sigma, \mathcal{G})$. The symplectic structure on $\operatorname{Hom}\left(\pi_{1}(\Sigma), G\right) / G$ descends from the symplectic structure on the infinite-dimensional space $\mathcal{A}$ of all connections on a fixed principal $G$-bundle on $\Sigma$. 
Such symplectic structure is simply given by the formula

$$
\omega(\alpha, \beta)=-\int_{\Sigma} K(\alpha \wedge \beta),
$$

where $K$ represents the Killing formula on $\mathcal{G}$ (henceforth we will write $\operatorname{tr}$ for the Killing form).

The gauge group $H$ acts on $\mathcal{A}$ by gauge transformations preserving the symplectic form; we then have a (moment) map (via the isomorphism $\Omega^{0}(\Sigma, \mathcal{G})^{*}=\Omega^{2}(\Sigma, \mathcal{G})$ )

$$
\begin{aligned}
& \mathcal{A} \longrightarrow \operatorname{Lie}(H)^{*}=\Omega^{0}(\Sigma, \mathcal{G})^{*}, \\
& A \longmapsto F_{A},
\end{aligned}
$$

where $F_{A}=$ curvature of $A$, inducing the following diagram:

$$
\begin{array}{rll}
\mathcal{A} & \stackrel{\mu}{\longrightarrow} & \operatorname{Lie}(H) \\
\downarrow & & \downarrow \\
\mathcal{A}_{\text {flat }} & \mapsto & 0
\end{array}
$$

where $\mathcal{A}_{\text {flat }}$ is then identified with the pre-image of zero via the map $\mu$ and it represents the set of flat connections.

By the Marsden-Weinstein theorem, $\mu^{-1}(0) / H=\operatorname{Hom}\left(\pi_{1}(\Sigma), G\right) / G$ has a symplectic structure induced by $\omega$ on $\mathcal{A}$.

$\omega$ acts on the second exterior power $\bigwedge^{2} T\left(\operatorname{Hom}\left(\pi_{1}(\Sigma), G\right) / G\right)$ of the tangent space $H_{A}^{1}(\Sigma, \mathcal{G})=\mathrm{Z}_{A}^{1}(\Sigma, \mathcal{G}) / B_{A}^{1}(\Sigma, \mathcal{G})$.

The symplectic form $\omega$ is then a 2 -form

$$
\omega: \bigwedge^{2}\left(H_{A}^{1}(\Sigma, \mathcal{G})\right) \rightarrow \mathbf{R}
$$

defined by

$$
\omega(\alpha, \beta)=-\int_{\Sigma} \operatorname{tr}(\alpha \wedge \beta)
$$

where $t r$ represents the trace (or, more in general, the Killing form $K$ ).

\section{THE SYMPLECTIC FORM}

We remind the reader that a Riemann surface is topologically determined by its genus and by the number of its boundary components.

Throughout this paper we will denote with $g$ the genus and with $n$ the number of boundary components unless otherwise specified; then the fundamental group will be presented by $(2 g+n)$ generators.

$\Sigma(g, n)$ will symbolise such a Riemann surface.

Also, we will generally call $a_{*}, b_{*}$ the $2 g$ generators corresponding to the genus (the $g$ "handles") and $c_{*}$ the $n$ generators corresponding to the boundary components.

Following this convention, we can prescribe the fundamental group by ([Arm83])

$$
\pi_{1}(\Sigma)=\left\{a_{1}, \ldots, a_{g}, b_{1}, \ldots, b_{g}, c_{1}, \ldots, c_{n} \mid \prod_{j=1}^{g}\left[a_{j}, b_{j}\right] \prod_{k=1}^{n} c_{k}=i d\right\} .
$$

Here $\left[a_{j}, b_{j}\right]$ represents the commutator of $a_{j}$ and $b_{j}$, and $i d$ represents the identity of the group. It should be noted that this group is not commutative and that the elements are multiplied in the order in which they are written. 
The fundamental group of $\Sigma(g, n)$ is actually a free group with $(2 g+n-1)$ generators, provided that $n>0$, but it is more convenient to think of it as a group generated by $(2 g+n)$ generators with 1 relation, at least to be able to mantain the same description also when $n=0$.

The relation

$$
R=\prod_{j=1}^{g}\left[a_{j}, b_{j}\right] \prod_{k=1}^{n} c_{k}=i d
$$

is topologically unique to the Riemann surface $\Sigma(g, n)$, and it will represent the base from which to construct the algebraic fundamental 2-chain associated with this surface.

In order to proceed with our algebraic re-interpretation of Riemann surfaces and of moduli spaces of structures on them, we need to introduce an important tool that we will use throughout this chapter, Fox calculus (see [Fox53] or [Gol84]).

The Fox calculus allows us to apply many operations performed in numerical calculus to elements of the fundamental group of a surface (for a complete treatment see [Fox53] or [Gol84]).

2.1. Fox calculus. Let $\Pi$ be a free group and $\mathbf{Z} \Pi$ be its integral group ring defined by $\mathbf{Z} \Pi=\left\{n_{i} p_{i} \mid n_{i} \in \mathbf{Z}, p_{i} \in \Pi\right\}$.

\section{Definition 2.1.1.}

$$
\epsilon: \begin{array}{ccc}
\mathbf{Z} \Pi & \rightarrow \mathbf{Z}, \\
\sum n_{i} p_{i} & \mapsto \sum n_{i},
\end{array}
$$

is called the augmentation homomorphism.

Definition 2.1.2. A derivation $D: \mathbf{Z} \Pi \rightarrow \mathbf{Z} \Pi$ is a $\mathbf{Z}$-linear map such that it satisfies the following "Leibniz" rule: $D\left(m_{1} m_{2}\right)=D\left(m_{1}\right) \epsilon\left(m_{2}\right)+m_{1} D\left(m_{2}\right)$, where $m_{1}, m_{2} \in \mathbf{Z} \Pi$ and $\epsilon$ is the augmentation homomorphism defined above.

Definition 2.1.3. If $p_{1}, \ldots, p_{n}$ is a set of generators for $\Pi$, we can define a set of derivations $\left\{\frac{\partial}{\partial p_{i}}\right\}$ satisfying $\frac{\partial}{\partial p_{i}}\left(p_{j}\right)=\delta_{i, j}$.

Lemma 2.1.4. $\frac{\partial}{\partial p}\left(p^{-1}\right)=-p^{-1}$

Lemma 2.1.5 (Mean Value Theorem). Let $p_{1}, \cdots, p_{n}$ be a set of generators for $\Pi$, then $\sum\left(\frac{\partial}{\partial p_{i}}(m)\right)\left(p_{i}-1\right)=m-\epsilon(m)$.

Proof. Both proofs are simple exercises.

Let $p_{1}, \cdots, p_{n}$ and $m$ be defined as in the previous proposition, and let $u: \Pi \rightarrow$ $\mathcal{G}_{A d \phi}$ be a 1-cocycle as in chapter 1, i.e. a map from $\Pi$ to $\mathcal{G}$ satisfying the cocycle condition $u(a b)=u(a)+\phi(a) u(b) \phi(a)^{-1}$.

Definition 2.1.6. For each 1-cocycle $u: \Pi \rightarrow \mathcal{G}_{A d \phi}$ we can define a new map $u: \mathbf{Z} \Pi \rightarrow \mathcal{G}_{A d \phi}$ by extending it by linearity and by the condition that, for elements $a, b$ of $\mathbf{Z} \Pi, u(a b)=u(a) \epsilon(b)+\phi(a) u(b) \phi(a)^{-1}$.

The action $\phi(a) u(b) \phi(a)^{-1}=A d_{\phi(a)} u(b)$ is also extended by linearity by the rule $A d_{\phi(n \alpha+m \beta)} u=n A d_{\phi(\alpha)} u+m A d_{\phi(\beta)} u$

\section{Proposition 2.1.7.}

$$
u(m)=\sum_{i} \phi\left(\frac{\partial}{\partial p_{i}}(m)\right) u\left(p_{i}\right) \phi\left(\frac{\partial}{\partial p_{i}}(m)\right)^{-1}
$$


Proof.

$$
\begin{aligned}
u(m) & =u(m-1)=u\left(\sum \frac{\partial}{\partial p_{i}}(m)\left(p_{i}-1\right)\right) \\
& =\sum u\left(\frac{\partial}{\partial p_{i}}(m)\right) \epsilon\left(p_{i}-1\right)+\sum \phi\left(\frac{\partial}{\partial p_{i}}(m)\right) u\left(p_{i}-1\right) \phi\left(\frac{\partial}{\partial p_{i}}(m)\right)^{-1} \\
& =\sum \phi\left(\frac{\partial}{\partial p_{i}}(m)\right) u\left(p_{i}\right) \phi\left(\frac{\partial}{\partial p_{i}}(m)\right)^{-1},
\end{aligned}
$$

since $\epsilon\left(p_{i}-1\right)=0$.

The purpose of this section is to use the Fox calculus to provide a simple expression for the 2-homology class corresponding to a Riemann surface. Any Riemann surface $\Sigma$ corresponds to a 2-chain; if the Riemann surface is closed, the 2-chain belongs to $Z^{2}(\Sigma)$. Since Riemann surfaces are Eilenberg-MacLane spaces, their (co-)homology spaces are isomorphic to their group (co-)homology spaces, in particular $H^{2}(\Sigma)=H^{2}\left(\pi_{1}(\Sigma)\right)$.

The spaces of $\mathrm{n}$-chains are $\mathbf{Z}$-modules freely generated by $\Pi \times \stackrel{n}{ } \cdot \times \Pi$; such spaces are denoted by $C_{n}\left(\pi_{1}(\Sigma)\right)$. On such spaces we can define a boundary operator $\delta$ between $n$-chains.

\section{Definition 2.1.8.}

$$
\begin{array}{ccl}
C_{n} & \stackrel{\delta}{\rightarrow} & C_{n-1}, \\
\left(z_{1}, \cdots, z_{n}\right) \stackrel{\delta}{\mapsto} & \epsilon\left(z_{1}\right)\left(z_{2}, \cdots, z_{n}\right)+(-1)^{n+1} \epsilon\left(z_{n}\right)\left(z_{1}, \cdots, z_{n-1}\right) \\
& +\sum_{1}^{n-1}(-1)^{j}\left(z_{1}, \cdots, z_{j} z_{j+1}, \cdots, z_{n}\right) .
\end{array}
$$

Since the boundary operator satisfies the condition $\delta^{2}=0$, it defines a long exact sequence in homology. For Riemann surfaces, the fundamental group $\pi_{1}(\Sigma)$ is a free group with one defining relation $R$, so we can use Hopf's formula ([Hop42], [Lyn50]). In particular, we have that to each relation $R$, given by products of commutators in the generators, there corresponds an associated homology class in $H^{2}\left(\pi_{1}(\Sigma)\right)$.

Goldman provides an explicit formula for the 2-cycle representing the Riemann surface whose homology class generates $H^{2}(\pi(\Sigma))$, such formula is given in the following definition.

Definition 2.1.9. The fundamental cycle of a Riemann surface $\Sigma$ for the relation

$$
R=\prod_{j=1}^{g}\left[a_{j}, b_{j}\right]=i d
$$

is given by

$$
\sum_{j=1}^{g}\left(\frac{\partial R}{\partial a_{j}}, a_{j}\right)+\sum_{j=1}^{g}\left(\frac{\partial R}{\partial b_{j}}, b_{j}\right) .
$$

This formula holds for closed Riemann surfaces. If we extend it to open Riemann surfaces, i.e. surfaces whose boundary is not empty, we obtain the following general formula: 
Definition 2.1.10. The fundamental 2-chain of a Riemann surface $\Sigma(g, n)$ for the relation

$$
R=\prod_{j=1}^{g}\left[a_{j}, b_{j}\right] \prod_{k=1}^{n} c_{k}=i d
$$

is given by

$$
\sum_{j=1}^{g}\left(\frac{\partial R}{\partial a_{j}}, a_{j}\right)+\sum_{j=1}^{g}\left(\frac{\partial R}{\partial b_{j}}, b_{j}\right)+\sum_{k=1}^{n}\left(\frac{\partial R}{\partial c_{k}}, c_{k}\right) .
$$

More generally, we can express both formulas by $\sum_{i=1}^{m}\left(\frac{\partial R}{\partial x_{i}}, x_{i}\right)$, where $\left\{x_{1}, \ldots, x_{m}\right\}$ represents the set of generators for the fundamental group and $R$ represents the relation (see [Lyn50] and [Gol84]). Using the rules for the Fox calculus, we can explicitly expand such a fundamental 2-chain. In order to do that we will evaluate $\frac{\partial R}{\partial a_{j}}, \frac{\partial R}{\partial b_{j}}$ and $\frac{\partial R}{\partial c_{k}}$ separately, and then we will re-write the resulting expression:

$$
\begin{aligned}
\frac{\partial R}{\partial a_{j}} & =\frac{\partial\left(\prod_{k=1}^{j-1}\left[a_{k}, b_{k}\right]\right)}{\partial a_{j}}+\prod_{k=1}^{j-1}\left[a_{k}, b_{k}\right] \frac{\partial\left(\prod_{k=j}^{g}\left[a_{k}, b_{k}\right] \prod_{k=1}^{n} c_{k}\right)}{\partial a_{j}} \\
& =\prod_{k=1}^{j-1}\left[a_{k}, b_{k}\right]\left\{1+a_{j} \frac{\partial\left(b_{j} a_{j}^{-1} b_{j}^{-1} \prod_{j+1}^{g}\left[a_{k}, b_{k}\right] \prod_{1}^{n} c_{k}\right)}{\partial a_{j}}\right\} \\
& =\prod_{k=1}^{j-1}\left[a_{k}, b_{k}\right]\left\{1+a_{j}\left(\frac{\partial b_{j}}{\partial a_{j}}+b_{j} \frac{\partial\left(a_{j}^{-1} b_{j}^{-1} \prod_{j+1}^{g}\left[a_{k}, b_{k}\right] \prod_{1}^{n} c_{k}\right)}{\partial a_{j}}\right)\right\} \\
& =\prod_{k=1}^{j-1}\left[a_{k}, b_{k}\right]\left\{1+a_{j}\left(b_{j} \frac{\partial\left(a_{j}^{-1}\right)}{\partial a_{j}}+a_{j}^{-1} \frac{\partial\left(b_{j}^{-1} \prod_{j+1}^{g}\left[a_{k}, b_{k}\right] \prod_{1}^{n} c_{k}\right)}{a_{j}}\right)\right\} \\
& =\prod_{k=1}^{j-1}\left[a_{k}, b_{k}\right]\left(1-a_{j} b_{j}\left(a_{j}^{-1}\right)\right) \\
& =\prod_{k=1}^{j-1}\left[a_{k}, b_{k}\right]\left(1-a_{j} b_{j} a_{j}^{-1}\right) .
\end{aligned}
$$

By repeating, mutatis mutandis, the same type of calculation, we can easily find that

$$
\frac{\partial R}{\partial b_{j}}=\prod_{k=1}^{j-1}\left[a_{k}, b_{k}\right]\left(a_{j}-a_{j} b_{j} a_{j}^{-1} b_{j}^{-1}\right)
$$

and

$$
\frac{\partial R}{\partial c_{j}}=\prod_{k=1}^{g}\left[a_{k}, b_{k}\right] \prod_{k=1}^{j-1} c_{k} .
$$

If we apply the definition of the boundary map $\delta$, given in its generality, to the case of 2-chains, the definition comes to:

Definition 2.1.11. The boundary operator on 2-chains is defined by

$$
\begin{array}{cl}
C_{2} & \longrightarrow C_{1}, \\
(u, v) & \mapsto \epsilon(u) v+\epsilon(v) u-u \cdot v .
\end{array}
$$


We shall use this definition and prove some important properties of the 2-chain we just defined. First of all we will describe explicitly what the action of the boundary operator is. When it acts on the fundamental 2-chain representing the Riemann surface, it produces a 1-chain, i.e. a sum of elements in the fundamental group of $\Sigma$.

It will soon be clear that, in the same way the fundamental 2-chain represents the Riemann surface, its boundary represents the "geometrical" boundary of the Riemann surface.

Since we can use the long exact sequence defined by the boundary operator on chains to define a homology of chains (elements in $H_{n}$ will be called $n$-cycles), it now appears clear that the fundamental 2-chain corresponding to a closed Riemann surface defines an element in $H_{2}$, i.e. it is actually a 2-cycle.

In fact:

$$
\begin{gathered}
\delta\left(\sum_{i=1}^{m}\left(\frac{\partial R}{\partial x_{i}}, x_{i}\right)\right)=\sum_{i=1}^{m} \epsilon\left(\frac{\partial R}{\partial x_{i}}\right) x_{i}+\epsilon\left(x_{i}\right) \frac{\partial R}{\partial x_{i}}-\frac{\partial R}{\partial x_{i}} \cdot x_{i} \\
=\epsilon\left(\frac{\partial R}{\partial x_{i}}\right) x_{i}+\sum_{i=1}^{m} \frac{\partial R}{\partial x_{i}} \cdot\left(\epsilon\left(x_{i}\right)-x_{i}\right)=\epsilon\left(\frac{\partial R}{\partial x_{i}}\right) x_{i}+1-R=\epsilon\left(\frac{\partial R}{\partial x_{i}}\right) x_{i}
\end{gathered}
$$

by the mean value theorem and the fact that $R=1$.

$\sum \epsilon\left(\frac{\partial R}{\partial x_{i}}\right) x_{i}$ then represents the boundary of the fundamental 2-chain, and we can evaluate the value of $\epsilon\left(\frac{\partial R}{\partial x_{i}}\right)$. We have already evaluated the possible expressions for $\frac{\partial R}{\partial x_{j}}$ in the three possible cases: when $x_{j}=a_{j}, b_{j}$ or $c_{j}$.

If $x_{j}=a_{j}$, then we get

$$
\frac{\partial R}{\partial x_{j}}=\prod_{1}^{j-1}\left[a_{k}, b_{k}\right]\left(1-a_{j} b_{j} a_{j}^{-1}\right)
$$

and

$$
\epsilon\left(\prod_{1}^{j-1}\left[a_{k}, b_{k}\right]\left(1-a_{j} b_{j} a_{j}^{-1}\right)\right)=0 .
$$

Similarly,

$$
\epsilon\left(\prod_{1}^{j-1}\left[a_{k}, b_{k}\right]\left(a_{j}-a_{j} b_{j} a_{j}^{-1} b_{j}^{-1}\right)\right)=0
$$

and

$$
\epsilon\left(\prod_{1}^{g}\left[a_{k}, b_{k}\right] \prod_{1}^{j-1} c_{k}\right)=1 .
$$

Therefore $\epsilon\left(\frac{\partial R}{\partial x_{i}}\right) x_{i}=\sum\left(c_{i}\right)$, where the sum is only taken over the elements " $c_{i}$ " representing the (geometrical) boundary of $\Sigma$.

Clearly we then have (geometrical) boundary $=$ (algebraic) boundary. The main point here is that, because $\frac{\partial\left(X^{-1}\right)}{\partial X}=-X^{-1}$, whenever $X$ represents a generator NOT on the boundary, the derivation of the relation $R$ with respect to it has two terms with opposite sign; hence its augmentation $\epsilon$ is zero. Otherwise, the derivation $\frac{\partial R}{\partial X}$ has only one term and its augmentation is 1 . The symplectic form can be defined in this algebraic context similarly to how it is geometrically defined. 
Let us remind the reader that the symplectic form on the space $X$ of $G$-connections on the space of stable bundles over a Riemann surface is defined by

$$
\omega(u, v)=-\int_{\Sigma} \operatorname{trace}(u \wedge v),
$$

where $u$ and $v$ represent the connection 1-forms (see [AB82]).

Similarly, we can define the symplectic form by

$$
\omega(u, v)=-\sum_{i} \operatorname{trace}\left(u\left(\# \frac{\partial R}{\partial x_{i}}\right) \cdot v\left(x_{i}\right)\right),
$$

where the sum is taken over all the generators, $u$ and $v$ represent 1-cocycles and \# represents the extension to the integer ring of the inverse, i.e. $\#(n \gamma+m \eta)=$ $n \gamma^{-1}+m \eta^{-1} ; \gamma, \eta \in \pi_{1}(\Sigma)$ (see [Gol84], [AM95] and [Kar92]). (Let us note in passing that in general we should replace the "trace" with the invariant inner product defined on the Lie algebra $\mathcal{G}$ of $G$; however, the trace is the right invariant inner product for both $\operatorname{PSL}(2, \mathbf{R})$ and $\operatorname{PGL}(3, \mathbf{R})$, and for $\operatorname{GL}(n, \mathbf{R})$ and $\operatorname{SL}(n, \mathbf{R})$ in general.)

Let us understand the analogy between the two formulas.

Clearly, the wedge product is replaced by the product of matrices, the invariant inner product is the same, and the important point is that integrating over the Riemann surface is equivalent to evaluating the pair of 1-cocycles on the fundamental 2-cycle.

However, this algebraic approach has the advantage of allowing us to extend the definition to open Riemann surfaces by using their fundamental 2-chain.

Such an operation defines a bilinear form (of course we lose some of the properties of $\omega$, for example its being skew-symmetric) that we call the open pre-symplectic form relative to the open Riemann surface $\Sigma$. The construction of the symplectic form in this algebraic formulation in its most general setting goes as follows $([\operatorname{Kar} 92])$.

Let $\Pi$ be a finitely generated group, if we consider the space $G^{\Pi}$ of all maps $\{\Pi \rightarrow G\}$ in which $\operatorname{Hom}(\Pi, G)$ is contained, its tangent space $T_{\phi} G^{\Pi}$ is given by $\mathcal{G}^{\Pi}=\{\Pi \rightarrow \mathcal{G}\}$.

Then we can define a vector-valued tensor $\omega$ on $\mathrm{G}^{\Pi}$ by

$$
\begin{array}{ccccc}
\omega: T_{\phi} G^{\Pi} & \times & T_{\phi} G^{\Pi} & \rightarrow & C^{2}(\Pi, \mathbf{R}), \\
u & & v & \mapsto & \omega_{\phi}(u, v),
\end{array}
$$

where $\omega_{\phi}(u, v)(\gamma, \eta)=B\left(u(\gamma), A d_{\phi(\gamma)} v(\eta)\right)$ and $C^{2}(\Pi, \mathbf{R})=\mathbf{R}^{\Pi \times \Pi}=\{$ all maps: $\Pi \times$ $\Pi \rightarrow \mathbf{R}\}$.

If $\phi \in \operatorname{Hom}(\Pi, G)$, then the map

$$
A d_{\phi}: \Pi \rightarrow \operatorname{Aut}(\mathcal{G})
$$

is a homomorphism of groups, and

$$
\omega_{\phi}(u, v)=u \cup v
$$

actually defines a cup-product on $T_{\phi} G^{\Pi}=\mathcal{G}^{\Pi}$.

The restriction of $\omega$ to the tangent space

$$
T_{\phi} \operatorname{Hom}(\Pi, G)=Z^{1}(\Pi, G)=\left\{u: \Pi \rightarrow G \mid u(\gamma \eta)=u(\gamma)+A d_{\phi(\gamma)} v(\eta)\right\}
$$


gives a tensor with values in

$$
Z^{2}(\Pi, \mathbf{R})=\{\Gamma: \Pi \times \Pi \rightarrow \mathbf{R} \mid \Gamma(\gamma, \eta)-\Gamma(\eta, \zeta)-\Gamma(\gamma \eta, \zeta)+\Gamma(\gamma, \eta \zeta)=0\} .
$$

If we map

$$
Z^{2}(\Pi, \mathbf{R}) \rightarrow H^{2}(\Pi, \mathbf{R})=Z^{2}(\Pi, \mathbf{R}) / B^{2}(\Pi, \mathbf{R}),
$$

where $B^{2}(\Pi, \mathbf{R})=\left\{\Delta: \Pi \times \Pi \rightarrow \mathbf{R} \mid \Delta(\gamma, \eta)=\delta \Delta_{0}(\gamma, \eta)=\Delta_{0}(\gamma)+\Delta_{0}(\eta)-\Delta_{0}(\gamma \eta)\right.$, for some $\left.\Delta_{0} \in Z^{1}(\Pi, \mathbf{R})\right\}$, we obtain a 2 -form on $\operatorname{Hom}(\Pi, G)$ which is degenerate along the $G$-orbits.

Karshon ([Kar92]) proves that the composition

$$
T_{\phi} G^{\Pi} \times T_{\phi} G^{\Pi} \stackrel{\omega}{\rightarrow} C^{2}(\Pi, \mathbf{R}) \stackrel{f}{\rightarrow} \mathbf{R}
$$

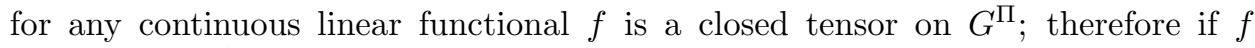
vanishes on $B^{2}(\Pi, \mathbf{R})$ we obtain a 2 -form which is degenerate along the $G$-orbits.

If these are the only degenerate directions, we obtain a symplectic structure on $\operatorname{Hom}(\Pi, G) / G$. Goldman defines the symplectic form by pairing the element in $H^{2}(\Pi, \mathbf{R})$ with the fundamental class of the Riemann surface in $H_{2}(\Pi)$, so defining the function $f$. Here, to define the open pre-symplectic form, we are pairing the element in $Z^{2}(\Pi, \mathbf{R})$ with an element in $Z_{2}(\Pi)$ representing the open Riemann surface.

We will now describe the properties of the open pre-symplectic form that we just defined.

2.2. Open pre-symplectic form properties. Many properties of the symplectic form on the moduli space of $G$-structures on a compact surface are well known. In particular, the symplectic form must be closed and skew-symmetric. Also, it acts on the cohomology space representing the tangent space of the space of representations modulo conjugation. None of those properties are kept by the open symplectic form $\omega$; however, we can define new ones.

First of all we will consider the (possible) skew-symmetry of $\omega$.

We will be studying the expression $\omega(u, v)+\omega(v, u)$. For the sake of brevity we use $\partial_{i}$ to denote $\frac{\partial}{\partial x_{i}}$.

By definition,

$$
\omega(u, v)=-\sum \operatorname{tr}\left(u\left(\# \partial_{i} R\right) \cdot v\left(x_{j}\right)\right)=-\sum \operatorname{tr}\left(A d_{\phi}\left(\partial_{i} \# \partial_{j} R\right) u\left(x_{i}\right) \cdot v\left(x_{j}\right)\right)
$$

and

$$
\begin{aligned}
\omega(v, u) & =-\sum \operatorname{tr}\left(A d_{\phi}\left(\partial_{j} \# \partial_{i} R\right) v\left(x_{j}\right) \cdot u\left(x_{i}\right)\right) \\
& =-\sum \operatorname{tr}\left(u\left(x_{i}\right) \cdot A d_{\phi}\left(\partial_{j} \# \partial_{i} R\right) v\left(x_{j}\right)\right) \\
& =-\sum \operatorname{tr}\left(A d_{\phi}\left(\# \partial_{j} \# \partial_{i} R\right) u\left(x_{i}\right) \cdot v\left(x_{j}\right)\right),
\end{aligned}
$$

so that

$$
\omega(u, v)+\omega(v, u)=-\sum \operatorname{trace}\left(A d_{\phi}\left(\left(\partial_{i} \# \partial_{j}+\# \partial_{j} \# \partial_{i}\right) R\right) u\left(x_{i}\right) \cdot v\left(x_{j}\right)\right) .
$$

Lemma 2.2.1. If $i \neq j$, then

$$
\partial_{i} \# \partial_{j} R+\# \partial_{j} \# \partial_{i} R=\left(\# \partial_{j} R\right)\left(\partial_{i} R\right)
$$

(The statement of this lemma, for closed surfaces, was suggested by R. Brown.) 
Proof. To fix the ideas let us suppose $R=\prod X_{k}$, where $X_{k}$ can represent either the commutator $\left[\mathrm{a}_{k}, \mathrm{~b}_{k}\right]$ or the boundary element $\mathrm{c}_{k}$.

Let

$$
\aleph_{j}^{i}= \begin{cases}1 & \text { if } i>j \\ 0 & \text { if } j>i .\end{cases}
$$

Then

$$
\begin{aligned}
\partial_{i} \# \partial_{j} R & =\partial_{i} \#\left[\left(\prod_{1}^{j-1} X_{k}\right)\left(\partial_{j} X_{j}\right)\right]=\partial_{i}\left[\left(\# \partial_{j} X_{j}\right)\left(\# \prod_{1}^{j-1} X_{k}\right)\right] \\
& =\#\left(\partial_{j} X_{j}\right)\left(\prod_{j-1}^{i} X_{k}^{-1}\right)\left(\partial_{i} X_{i}\right) \aleph_{i}^{j}
\end{aligned}
$$

so that

$$
\partial_{i} \# \partial_{j} R+\# \partial_{j} \# \partial_{i} R=\left(\partial_{j} X_{j}\right)^{-1}\left(\prod_{j-1}^{i} X_{k}^{-1}\right)\left(\partial_{i} X_{i}\right) \aleph_{i}^{j}+\left(\partial_{j} X_{j}\right)^{-1}\left(\prod_{j}^{i-1} X_{k}\right)\left(\partial_{i} X_{i}\right) \aleph_{j}^{i} .
$$

On the other hand,

$$
\begin{gathered}
\left.\left(\# \partial_{j} R\right)\left(\partial_{i} R\right)=\left(\#\left(\partial_{j} X_{j}\right)\right)\left(\prod_{j-1}^{1} X_{k}^{-1}\right)\right)\left(\left(\prod_{1}^{i-1} X_{k}\right)\left(\partial_{i} X_{i}\right)\right) \\
=\#\left(\partial_{j} X_{j}\right)\left(\prod_{j-1}^{i} X_{k}^{-1}\right)\left(\partial_{i} X_{i}\right) \aleph_{i}^{j}+\#\left(\partial_{j} X_{j}\right)\left(\prod_{j}^{i-1} X_{k}\right)\left(\partial_{i} X_{i}\right) \aleph_{j}^{i} .
\end{gathered}
$$

Whenever $i=j$ we have the following:

$$
\begin{gathered}
\partial_{i} \# \partial_{i} R+\# \partial_{i} \# \partial_{i} R=\partial_{i} \#\left(\left(\prod_{1}^{i-1} X_{k}\right)\left(\partial_{i} X_{i}\right)\right)+\# \partial_{i} \#\left(\left(\prod_{1}^{i-1} X_{k}\right)\left(\partial_{i} X_{i}\right)\right) \\
\left.\left.=\partial_{i}\left(\#\left(\partial_{i} X_{i}\right) \#\left(\prod_{1}^{i-1} X_{k}\right)\right)\right)+\# \partial_{i}\left(\#\left(\partial_{i} X_{i}\right) \#\left(\prod_{1}^{i-1} X_{k}\right)\right)\right)
\end{gathered}
$$

and

$$
\left(\# \partial_{i} R\right)\left(\partial_{i} R\right)=\left(\#\left(\partial_{i} X_{i}\right)\right)\left(\partial_{i} X_{i}\right)
$$

so that the two terms are equal if $\partial_{i}\left(\partial_{i} X_{i}\right)=-\partial_{i} X_{i}$ as is the case if $X_{i}=\left[a_{i}, b_{i}\right]$ (as an easy calculation can show).

If $X_{i}=c_{i}$ it is easy to check that $\partial_{i} \# \partial_{i} R+\# \partial_{i} \# \partial_{i} R=0$ and $\left(\# \partial_{j} R\right)\left(\partial_{i} R\right)=1$. Therefore

$$
\begin{aligned}
\omega(u, v) & +\omega(v, u) \\
= & -\sum_{i, j} \operatorname{tr}\left(A d_{\phi}\left(\left(\partial_{i} \# \partial_{j}+\# \partial_{j} \# \partial_{i}\right) R\right) u\left(x_{i}\right) \cdot v\left(x_{j}\right)\right) \\
= & -\sum_{i, j} \operatorname{tr}\left(A d_{\phi}\left(\left(\# \partial_{j} R\right)\left(\partial_{i} R\right)\right) u\left(x_{i}\right) \cdot v\left(x_{j}\right)\right)+\sum_{x_{j} \in b d r y} \operatorname{tr}\left(u\left(x_{j}\right), v\left(x_{j}\right)\right) \\
= & -\operatorname{trace}(u(R) \cdot v(R))+\sum_{\text {bdry terms }} \operatorname{trace}\left(u\left(x_{j}\right) \cdot v\left(x_{j}\right)\right) .
\end{aligned}
$$


Theorem 2.2.2. $\omega$ is a skew-symmetric tensor for Riemann surfaces with no boundary. Otherwise

$$
\omega(u, v)+\omega(v, u)=\sum_{b d r y \text { terms }} \operatorname{trace}\left(u\left(x_{j}\right), v\left(x_{j}\right)\right) .
$$

Another important property of the symplectic form is that it is defined on homology classes, i.e. it is zero when evaluated on exact 1-cocycles.

Let $u_{0}$ be a 0 -cocycle, so that the exact 1 -cocycle $d u_{0}=u_{0}-A d_{\phi} u_{0}$. Then

$$
\begin{aligned}
& \omega\left(d u_{0}, v\right)=-\sum_{i} \operatorname{trace}\left(\left(d u_{0}\left(\# \frac{\partial R}{\partial x_{i}}\right) \cdot v\left(x_{i}\right)\right)\right. \\
& =-\sum_{i} \operatorname{trace}\left(\epsilon\left(\# \frac{\partial R}{\partial x_{i}}\right) u_{0} \cdot v\left(x_{i}\right)\right)+\sum_{i} \operatorname{trace}\left(A d_{\phi}\left(\# \frac{\partial R}{\partial x_{i}}\right) u_{0} \cdot v\left(x_{i}\right)\right) \\
& \left.=-\sum_{b d r y \text { terms }} \operatorname{trace}\left(u_{0} \cdot v\left(x_{i}\right)\right)+\sum_{i} \operatorname{trace}\left(u_{0}\right) \cdot A d_{\phi}\left(\frac{\partial R}{\partial x_{i}}\right) v\left(x_{i}\right)\right) \\
& =-\operatorname{trace}\left(u_{0} \cdot v\left(\sum_{b d r y}\left(x_{i}\right)\right)+\operatorname{trace}\left(u_{0} \cdot v(R)\right) .\right.
\end{aligned}
$$

Since clearly $v(R)=0$, we have

Theorem 2.2.3.

$$
\omega\left(d u_{0}, v\right)=-\operatorname{trace}\left(u_{0}, v(\text { boundary })\right) .
$$

This is basically Stokes's theorem in this algebraic context; in fact, we can rewrite the previous equality as

$$
\left.\omega\left(d u_{0}, v\right)\right|_{F C}=\left.\omega\left(u_{0}, v\right)\right|_{\delta(F C)}
$$

where $F C$ and $\delta F C$ represent the fundamental chain of the Riemann surface and its boundary, respectively. Therefore $\omega\left(d u_{0}, v\right)=0$ if and only if $\Sigma$ has no boundary. Using the fact that $\omega$ is skew-symmetric on a closed surface, that also proves that $\omega\left(u, d v_{0}\right)=0$.

2.3. "Algebraic" glueing. What we are interested in is to find an algebraic ana$\log$ of the connected sum (or topological glueing of boundary components) of two Riemann surfaces for fundamental 2-chains using group amalgamation.

There are two different ways to glue boundary components together: the first one deals with boundary components of two different Riemann surfaces; the second (HNN-glueing) deals with two different boundary components from the same Riemann surface.

We are interested in both cases, but for some of our purposes it will be more involved to deal with the HNN-glueing.

2.3.1. Connected glueing. For the sake of clarity let us fix two Riemann surfaces $\Sigma(g, n)$ and $\Sigma^{\prime}\left(g^{\prime}, n^{\prime}\right)$ with relations $R=i d$ and $R^{\prime}=i d$ respectively, and for the sake of simplicity (and with no loss of generality, since the definitions are independent of the presentation chosen) let us assume that we are glueing the boundary components represented by the terms $c_{n}$ and $c_{n^{\prime}}^{\prime}$.

Connected glueing means identifying those two boundary components, albeit with their respective orientation reversed (we are dealing with oriented Riemann surfaces and we are requiring the new Riemann surface to be oriented as well, for 


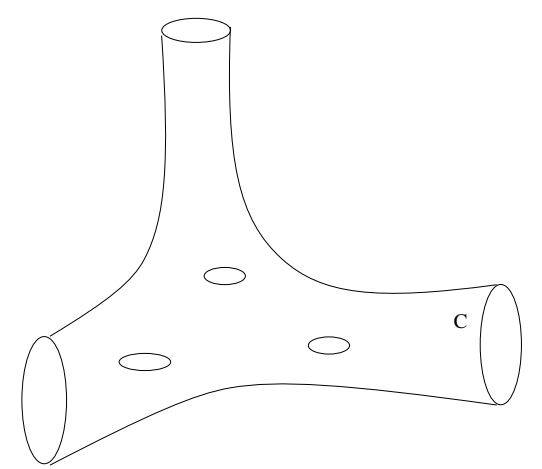

Riemann surface with relation $\mathrm{Rc}=\mathrm{id}$

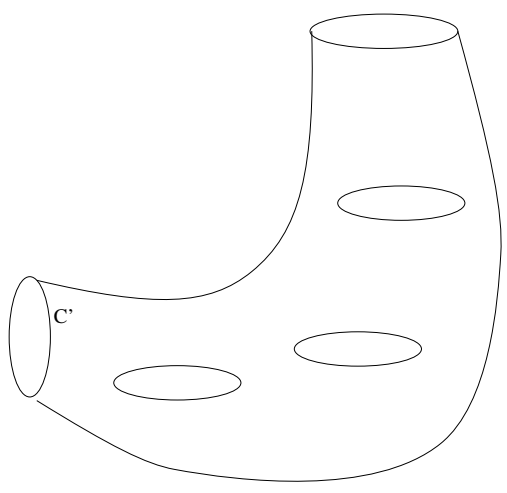

Riemann surface with relation R'c'=id

FiguRE 1. Connected glueing of two Riemann surfaces

which we need the boundary components to have opposite orientations); that is, we are introducing the group relation $c_{n}=c_{n^{\prime}}^{\prime-1}$ in the group freely generated by the pair (see Figure 1).

In the same way that we can "geometrically" glue two Riemann surfaces together, we can provide an "algebraic" way to glue their corresponding fundamental 2chains.

Glueing two Riemann surfaces $\Sigma(g, n)$ and $\Sigma^{\prime}\left(g^{\prime}, n^{\prime}\right)$ together, we get a new Riemann surface $\Sigma\left(g+g^{\prime}, n+n^{\prime}-2\right)$ whose fundamental cycle is given by

$$
\begin{gathered}
F C\left(\Sigma\left(g+g^{\prime}, n+n^{\prime}-2\right)\right) \\
=\sum_{j=1}^{g+g^{\prime}}\left\{\left(\prod_{k=1}^{j-1}\left[a_{k}, b_{k}\right]\left(1-a_{j} b_{j} a_{j}^{-1}\right), a_{j}\right)+\left(\prod_{k=1}^{j-1}\left[a_{k}, b_{k}\right]\left(a_{j}-a_{j} b_{j} a_{j}^{-1} b_{j}^{-1}\right), b_{j}\right)\right\} \\
+\sum_{j=1}^{n+n^{\prime}-2}\left(\prod_{1}^{g}\left[a_{k}, b_{k}\right] \prod_{1}^{j-1} c_{k}, c_{j}\right) .
\end{gathered}
$$

Given the fundamental chains $F C(\Sigma)$ for $\Sigma(g, n)$ and $F C\left(\Sigma^{\prime}\right)$ for $\Sigma^{\prime}\left(g^{\prime}, n^{\prime}\right)$, we shall then provide a formula on how to "glue" them together to obtain the fundamental chain for $\Sigma\left(g+g^{\prime}, n+n^{\prime}-2\right)$.

Let $R_{1} c_{1}=i d$ and $R_{2} c_{2}=i d$ be the relations relative to the fundamental groups $\pi_{1}\left(\Sigma_{1}\right)$ and $\pi_{1}\left(\Sigma_{2}\right)$ respectively, and let us suppose that we are identifying $c_{1}$ with $c_{2}^{-1}$. Let us recall that we can briefly describe the formula for the fundamental 2-chain for $\Sigma_{1}$ and $\Sigma_{2}$ by

$$
F C\left(\Sigma_{1}\right)=\sum_{i=1}^{g+n}\left(\frac{\partial\left(R_{1} c_{1}\right)}{\partial x_{i}}, x_{i}\right) \text { and } F C\left(\Sigma_{2}\right)=\sum_{i=1}^{g^{\prime}+n^{\prime}}\left(\frac{\partial\left(R_{2} c_{2}\right)}{\partial y_{j}}, y_{j}\right),
$$

where $\left\{x_{i}\right\}$ and $\left\{y_{j}\right\}$ are the sets of generators for $\Sigma$ and $\Sigma^{\prime}$. 
The first important observation is that (from the Leibniz rule),

so that we can write

$$
\begin{cases}\frac{\partial(R c)}{\partial x_{i}}=\frac{\partial R}{\partial x_{i}} & \text { if } x_{i} \neq c \\ \frac{\partial(R c)}{\partial c}=R & \text { if } x_{i}=c\end{cases}
$$

$$
F C\left(\Sigma_{1}\right)=\sum_{i=1}^{g+n-1}\left(\frac{\partial R_{1}}{\partial x_{i}}, x_{i}\right)+\left(R_{1}, c_{1}\right)
$$

and

$$
F C\left(\Sigma_{2}\right)=\sum_{j=1}^{g^{\prime}+n^{\prime}-1}\left(\frac{\partial R_{2}}{\partial y_{j}}, y_{j}\right)+\left(R_{2}, c_{2}\right)
$$

The second observation is that the defining relation for $\pi_{1}\left(\Sigma_{1} \# \Sigma_{2}\right)$ (here \# means "connected glueing"), is given by $R_{1} R_{2}=i d$, since $R_{1} c_{1}=i d=R_{2} c_{2}$ and $c_{1}=c_{2}^{-1} \Longrightarrow R_{1} R_{2}=i d$.

Therefore

$$
F C\left(\Sigma_{1} \# \Sigma_{2}\right)=\left(\sum_{i=1}^{g+n-1} \frac{\partial\left(R_{1} R_{2}\right)}{\partial x_{i}}, x_{i}\right)+\left(\sum_{j=1}^{g^{\prime}+n^{\prime}-1} \frac{\partial\left(R_{1} R_{2}\right)}{\partial y_{j}}, y_{j}\right) .
$$

By using "the product formula" for Fox calculus derivatives, we get

$$
\begin{aligned}
F C\left(\Sigma_{1} \# \Sigma_{2}\right) & =\left(\sum_{i=1}^{g+n-1} \frac{\partial R_{1}}{\partial x_{i}}, x_{i}\right)+\left(\sum_{j=1}^{g^{\prime}+n^{\prime}-1} \frac{R_{1} \partial R_{2}}{\partial y_{j}}, y_{j}\right) \\
& =F C\left(\Sigma_{1}\right)-\left(R_{1}, c_{1}\right)+\left(\sum_{j=1}^{g^{\prime}+n^{\prime}-1} \frac{R_{1} \partial R_{2}}{\partial y_{j}}, y_{j}\right) .
\end{aligned}
$$

Definition 2.3.1. For $R$ in $G$ we define a chain operator $\Phi_{R}$ on fundamental 2chains by

$$
\Phi_{R}\left(\sum\left(A_{i}, B_{i}\right)\right)=\sum\left(R A_{i}, B_{i}\right)
$$

Theorem 2.3.2. Given two Riemann surfaces $\Sigma_{1}(g, n)$ and $\Sigma_{2}\left(g^{\prime}, n^{\prime}\right)$ and their connected glueing surface $\Sigma\left(g+g^{\prime}, n+n^{\prime}-2\right)$, their fundamental chains are related by the formula

$$
F C(\Sigma)=F C\left(\Sigma_{1}\right)+\Phi_{R_{1}}\left(F C\left(\Sigma_{2}\right)\right)-\left(R_{1}, c_{1}\right)-\left(R_{1} R_{2}, c_{2}\right)
$$

where $R_{1} c_{1}=i d$, and $R_{2} c_{2}=i d$ represent the relations for the fundamental group of $\Sigma_{1}$ and $\Sigma_{2}$ respectively, and $\Phi_{R_{1}}$ is the chain operator.

2.3.2. HNN-glueing. Similarly to how we have dealt with glueing of boundary components of two different Riemann surfaces, we now study how the fundamental chain is affected by glueing two boundary components of the same Riemann surface.

Let $\Sigma$ be a Riemann surface and $R c_{1} c_{2}=i d$ the relation relative to its fundamental group. We will now suppose we are glueing the two boundary components whose corresponding elements in $\pi_{1}(\Sigma)$ are $c_{1}$ and $c_{2}$. Again, because of the independence of the Riemann surface from the presentation chosen, we can assume, without loss of generality, that this is the general case.

Let us suppose that $x$ is the path connecting $c_{1}$ and $c_{2}$. 


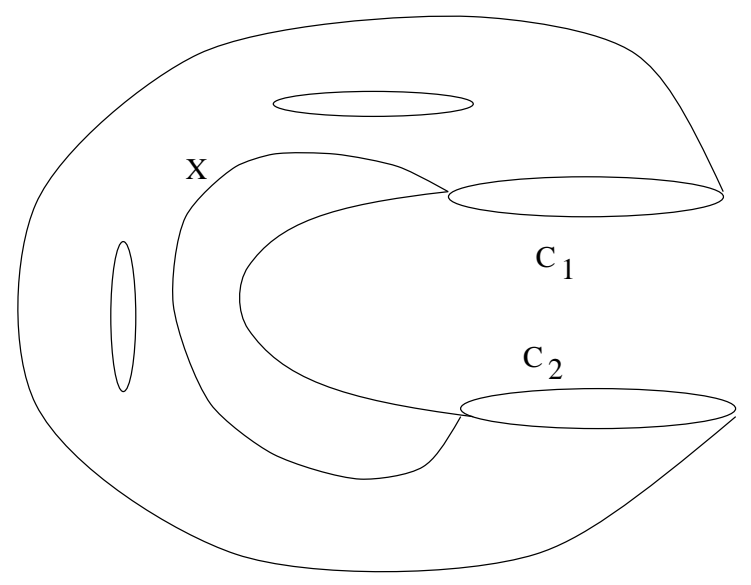

Figure 2. HNN-glueing of a Riemann surface.

Identifying the two boundary components relative to $c_{1}$ and $c_{2}$ is then equivalent to introducing the relation $c_{2}=x c_{1}^{-1} x^{-1}$ (see Figure 2).

The relation for the new fundamental group $\pi_{1}(\hat{\Sigma})$ is then given by the following: $R c_{1} x c_{1}^{-1} x^{-1}=i d$.

Observation: By the "product formula" for Fox derivatives, it is clear that $\frac{\partial(A B)}{\partial u}=\frac{\partial A}{\partial u}$ if the generator $u$ does not appear in the element $B$.

Therefore we have

Theorem 2.3.3. Let $\Sigma$ be a Riemann surface and $\hat{\Sigma}$ the resulting Riemann surface after having glued two boundary components of $\Sigma$.

The fundamental chains for the two Riemann surfaces are related by

$$
F C(\hat{\Sigma})=F C(\Sigma)-\left(R c_{1}, c_{2}\right)+\left(R c_{1}, x\right)-(1, x)-\left(x, c_{1}\right) .
$$

Proof. By the previous observation and simple Fox-derivations it is clear that

$$
\begin{cases}\frac{\partial\left(R c_{1} c_{2}\right)}{\partial u}=\frac{\partial\left(R\left[c_{1}, x\right]\right)}{\partial u}, & \text { if } u \neq c_{1}, c_{2} \text { or } x, \\ \frac{\partial\left(R c_{1} c_{2}\right)}{\partial c_{1}}=R, & \\ \frac{\partial\left(R\left[c_{1}, x\right]\right.}{\partial c_{1}}=R-R c_{1} x c_{1}^{-1}=R-x, & \\ \frac{\partial\left(R c_{1} c_{2}\right)}{\partial c_{2}}=R c_{1}, & \\ \frac{\partial\left(R\left[c_{1}, x\right]\right)}{\partial x}=R c_{1}-1 . & \end{cases}
$$

By simply substituting the corresponding terms in the formula for the fundamental chain we prove the statement.

2.4. Symplectic form glueing. As for the fundamental cycle, we will deal with both types of glueing, applying the formulae found in the previous section.

The general formula for the symplectic form

$$
\omega(u, v)=-\sum_{i} \operatorname{trace}\left(u\left(\# \frac{\partial R}{\partial x_{i}}\right) \cdot v\left(x_{i}\right)\right)
$$

for a generic Riemann surface $\Sigma(g, n)$ then becomes 


$$
\begin{gathered}
\omega(u, v)=-\sum_{j} \operatorname{trace}\left(u\left(\#\left(\prod_{k=1}^{j-1}\left[a_{k}, b_{k}\right]\left(1-a_{j} b_{j} a_{j}^{-1}\right)\right) \cdot v\left(a_{j}\right)\right)\right. \\
-\sum_{j} \operatorname{trace}\left(u\left(\#\left(\prod_{k=1}^{j-1}\left[a_{k}, b_{k}\right]\left(a_{j}-a_{j} b_{j} a_{j}^{-1} b_{j}^{-1}\right)\right) \cdot v\left(b_{j}\right)\right)\right. \\
+\sum_{j} \operatorname{trace}\left(u\left(\#\left(\prod_{k=1}^{g}\left[a_{k}, b_{k}\right] \prod_{k=1}^{j-1} c_{k}\right)\right) \cdot v\left(c_{j}\right)\right) .
\end{gathered}
$$

We will proceed analogously to the way we did in the previous section by considering two Riemann surfaces with relations $R_{1} c_{1}=i d$, and $R_{2} c_{2}=i d$, and their connected glueing surface with relation $R_{1} R_{2}=i d$.

For the connected glueing the general formula is

$$
\begin{gathered}
\omega(u, v)=\sum_{i} \operatorname{trace}\left(u\left(\# \frac{\partial\left(R_{1} R_{2}\right)}{\partial x_{i}}\right) \cdot v\left(x_{i}\right)\right)+\sum_{j} \operatorname{trace}\left(u\left(\# \frac{\partial\left(R_{1} R_{2}\right)}{\partial y_{j}}\right) \cdot v\left(y_{j}\right)\right) \\
=\sum_{i} \operatorname{trace}\left(u\left(\# \frac{\partial R_{1}}{\partial x_{i}}\right) \cdot v\left(x_{i}\right)\right)+\sum_{j} \operatorname{trace}\left(u\left(\#\left(R_{1} \frac{\partial R_{2}}{\partial y_{i}}\right)\right) \cdot v\left(y_{i}\right)\right) \\
=\omega_{1}(u, v)+\sum_{j} \operatorname{trace}\left(u\left(\# R_{1} \frac{\partial R_{2}}{\partial y_{i}}\right) \cdot v\left(y_{i}\right)\right)
\end{gathered}
$$

by the same reasoning as in the previous section (here $\omega_{1}$ represents the "formal" expression for the symplectic form on the Riemann surface with relation $R_{1} c_{1}$ ).

The second term in the previous expression can be rewritten as

$$
\begin{aligned}
\sum_{j} \operatorname{trace}\left(u\left(\# \frac{\partial R_{2}}{\partial y_{j}} \# R_{1}\right) \cdot v\left(y_{j}\right)\right) \\
\quad=\sum_{j} \operatorname{trace}\left(u\left(\# \frac{\partial R_{2}}{\partial y_{j}}\right) \cdot v\left(y_{j}\right)\right)+\sum_{j} \operatorname{trace}\left(\# \frac{\partial R_{2}}{\partial y_{j}} \cdot u\left(\# R_{1}\right) \cdot \frac{\partial R_{2}}{\partial y_{j}} \cdot v\left(y_{j}\right)\right) \\
\quad=\omega_{2}+\sum_{j} \operatorname{trace}\left(\# \frac{\partial R_{2}}{\partial y_{j}} \cdot u\left(\# R_{1}\right) \cdot \frac{\partial R_{2}}{\partial y_{j}} \cdot v\left(y_{j}\right)\right) \\
\quad=\omega_{2}+\sum_{j} \operatorname{trace}\left(u\left(\# R_{1}\right) \cdot \frac{\partial R_{2}}{\partial y_{j}} \cdot v\left(y_{j}\right) \cdot \# \frac{\partial R_{2}}{\partial y_{j}}\right)=\omega_{2}+\operatorname{trace}\left(u\left(\# R_{1}\right) \cdot v\left(R_{2}\right)\right)
\end{aligned}
$$

(here $\omega_{2}$ represents the "formal" expression for the symplectic form on the Riemann surface with relation $R_{2} c_{2}$ ).

Theorem 2.4.1. The symplectic form $\omega$ for a Riemann surface $\Sigma$, connected glueing of $\Sigma^{\prime}$ and $\Sigma^{\prime \prime}$, is related to the symplectic forms $\omega^{\prime}$ and $\omega^{\prime \prime}$ of $\Sigma^{\prime}$ and $\Sigma^{\prime \prime}$ by the formula

$$
\omega(u, v)=\omega^{\prime}(u, v)+\omega^{\prime \prime}(u, v)-\operatorname{trace}\left(u\left(c^{\prime}\right) \cdot v\left(c^{\prime \prime}-1\right)\right),
$$

where $c^{\prime}$ and $c^{\prime \prime}$ are the boundary components of $\Sigma^{\prime}$ and $\Sigma^{\prime \prime}$ respectively. 
This formula requires a more detailed analysis. In our formulation it says that, formally, the symplectic form for the connected glueing surface can be evaluated on the fundamental cycles of the two distinct Riemann surfaces.

That allows us to simplify some of the calculations.

We proceed now to determine how the symplectic form $\omega$ of a Riemann surface behaves under an HNN-glueing.

If $\Sigma$ is a Riemann surface with relation $R c_{1} c_{2}$ and $\hat{\Sigma}$ represents the resulting Riemann surface after having glued two boundary components of $\Sigma$, we proved that the fundamental chains of the two Riemann surfaces are related by the formula

$$
F C(\hat{\Sigma})=F C(\Sigma)-\left(R c_{1}, c_{2}\right)+\left(R c_{1}, x\right)-(1, x)-\left(x, c_{1}\right) .
$$

If we evaluate the symplectic formula $\omega$ on $F C(\hat{\Sigma})$, that is equivalent to evaluating it on $F C(\Sigma)-\left(R c_{1}, c_{2}\right)+\left(R c_{1}, x\right)-(1, x)-\left(x, c_{1}\right)$, which gives

Theorem 2.4.2. The symplectic form $\hat{\omega}$ of a Riemann surface $\hat{\Sigma}$ satisfies the following equality:

$$
\hat{\omega}(u, v)=\omega(u, v)-\operatorname{tr}(u(\# R x) \cdot v(\# R x))+\operatorname{tr}(u(\# R x) \cdot v(y))-\operatorname{tr}(u(\# y) \cdot v(x)),
$$

where $\omega$ represents the symplectic form of the surface before the glueing and $R[x, y]$ $=i d$ represents the relation relative to its fundamental group.

We can now make the following observation.

Let $\Sigma$ be a Riemann surface with one boundary component $c$ and relation $R c=$ $i d$. Let us call $\hat{\omega}$ its symplectic form and $\omega$ the symplectic form for the closed Riemann surface with relation $R=i d$.

Since

$$
\begin{cases}\frac{\partial(R c)}{\partial x_{i}}=\frac{\partial R}{\partial x_{i}} & \text { if } x_{i} \neq c \\ \frac{\partial R c}{\partial c}=R & \text { if } x_{i}=c\end{cases}
$$

then $\hat{\omega}(u, v)=\omega(u, v)+\operatorname{trace}(u(c) \cdot v(c))$.

Therefore, using the glueing formulae, we can find an explicit expression for the symplectic form of an open Riemann surface with one boundary component.

\section{PSL $(2, \mathbf{R})$ : THE HYPERBOLIC CASE AS EXAMPLE}

We will now turn our attention to the space of hyperbolic structures on a Riemann surface $\Sigma$. The deformation space, also called Teichmüller space, corresponds to an open subset of $\operatorname{Hom}\left(\pi_{1}(\Sigma), \operatorname{PSL}(2, \mathbf{R})\right) / \operatorname{PSL}(2, \mathbf{R})$, and its tangent space at the equivalence class of a representation $\Phi$ is given by the space of 1-cocycles $H^{1}\left(\pi_{1}(\Sigma), \mathcal{P} \mathcal{S} \mathcal{L}_{A d_{\Phi}}(2, \mathbf{R})\right)$.

We will reprove some of Wolpert's results ([Wol85]) about the symplectic form on this space by using the algebraic approach introduced in the previous sections.

3.1. The Fenchel-Nielsen coordinates. Some very useful coordinates are defined on Teichmüller space, the Fenchel-Nielsen coordinates.

Let us consider for example a trinion, i.e. a Riemann surface of genus zero (a sphere) and three boundary components, $\Sigma(0,3)$.

By general results of the classical theory of Riemann surfaces, the lengths of the loops defining the boundary components completely determine the hyperbolic structure on a pair of pants (a trinion). 
Moreover, the length of the loop corresponding to a boundary component is related to the trace of a matrix in the conjugacy class to which such loop is mapped in $\operatorname{PSL}(2, \mathbf{R})$; such relation is given by $l(\gamma)=2 \cosh ^{-1}\left(\left|\operatorname{tr} \frac{\Phi(\gamma)}{2}\right|\right)$. In fact, let us fix a trinion $P$ with relation $R=C B A=i d$, with $A, B, C$ denoting the loops representing the three boundary components. Such a description is purely topological, and we can impose a hyperbolic structure on $P$. A hyperbolic structure corresponds to a representation $\Phi$

$$
\left(\Phi: \pi_{1}(\Sigma) \rightarrow \operatorname{PSL}(2, \mathbf{R})\right) \bmod \operatorname{PSL}(2, \mathbf{R})
$$

which is completely determined by knowing $\Phi(A), \Phi(B)$ and $\Phi(C)$ since $A, B$ and $C$ are generators.

Note that, by abuse of notation, in the following we will denote $A, B, C$ both the boundary loops and the corresponding matrices $\phi(A), \phi(B), \phi(C)$ corresponding to a representation $\phi \in \operatorname{Hom}\left(\pi_{1}(\Sigma), G\right) / G$, wherever it is clear from the context what we are referring to.

In order to show that the lengths of the loops representing the boundary components determine the hyperbolic structure, we will prove that to fix the three matrices $\Phi(A), \Phi(B)$ and $\Phi(C)$ it is enough to know the traces of the matrices (modulo a common $\operatorname{PSL}(2, \mathbf{R})$-conjugation).

Just to fix the ideas we will count the number of expected free parameters that we have a priori. Since we are determining three 2 by 2 -matrices we have 12 free parameters. However the relation $C B A=i d$ completely determines $A$, knowing $C$ and $B$. That leaves us with 8 parameters; moreover, since the matrices have determinant 1 , there are only 6 free parameters.

Furthermore, since we are considering representations modulo $\operatorname{PSL}(2, \mathbf{R})$, the action of the group determines three more parameters, reducing the count of expected free parameters to 3 , which is exactly the number of variables we need, i.e. the three traces.

Definition 3.1.1. The Fenchel-Nielsen coordinates on a trinion are given by the lengths $l_{1}, l_{2}$ and $l_{3}$ of the boundary loops, or their algebraic counterpart, the absolute value of the traces of the matrices relative to the representation of those loops in $\operatorname{PSL}(2, \mathbf{R})$.

Whenever we build a Riemann surface, either by glueing trinions together or by operating HNN-glueings, we introduce a new degree of freedom. In fact, by rotating one boundary component with respect to another, we can operate the glueing differently. While the "twist" does not affect the Riemann surface topologically, it does change its hyperbolic structure.

More explicitly, we will discuss the case of an HNN-glueing and how to explicitly evaluate $\theta$, the Fenchel-Nielsen coordinate relative to the "twist" (for an exhaustive discussion of the Fenchel-Nielsen coordinates, see [Wol82] and [Wol88]).

3.2. The vector fields. Starting from the three coordinates $l_{1}, l_{2}$ and $l_{3}$, the hyperbolic traces of the three boundary components of the trinion, we are now going to construct a representation $\Phi$. The representation will be completely determined by its values on the generators $A$ and $B$. Since PSL $(2, \mathbf{R})$ acts by conjugation, we can assume that $A$ is a diagonal matrix. Let

$$
A=\left(\begin{array}{cc}
x & 0 \\
0 & \frac{1}{x}
\end{array}\right), B=\left(\begin{array}{cc}
a & b \\
c & d
\end{array}\right) .
$$


$C$ will be determined by the condition $A B C=i d$.

Also, because of the group action, we can choose one of the parameters in $B$ to be one, e.g. $b=1$. Note that $b \neq 0$, since otherwise $B(0)=\frac{a(0)+b}{c(0)+d}=0$, which is not possible in a discrete representation since we already have, because of the choices made, $A(0)=0$.

The conditions to impose are then

$$
\begin{gathered}
x+\frac{1}{x}=-\frac{1}{2} \cosh \left(2 l_{1}\right) ; \quad a+d=-\frac{1}{2} \cosh \left(2 l_{2}\right) ; \quad a x+\frac{d}{x}=-\frac{1}{2} \cosh \left(2 l_{3}\right), \\
a d-c=1 .
\end{gathered}
$$

Let us notice that, by general theory, we cannot have all three traces positive. The theory states that an odd number of traces must be negative ([GM91], [Har77]). Here we assume that all three traces are negative. That, in turn, imposes some obvious conditions on $x, a$ and $d$, i.e. that $x$ and $d$ be negative and $a$ positive. The system completely determines $x, a, c$ and $d$, and therefore the representation $\Phi$, knowing $l_{1}, l_{2}, l_{3}$.

In order to calculate the action of the vector fields $\frac{\partial}{\partial l_{*}}$ we first need to know the derivatives of $x, a$ and $d$ with respect to the $l_{*}$-coordinates, for which purpose we need to invert the matrix given by the jacobian of the derivatives of the $l_{*-}$ coordinates with respect to $x, a$ and $d$.

By definition,

$$
\begin{gathered}
l_{1}=\frac{1}{2} \cosh ^{-1}\left(2\left(x+\frac{1}{x}\right)\right) ; \quad l_{2}=\frac{1}{2} \cosh ^{-1}(2(a+d)) ; \\
l_{3}=\frac{1}{2} \cosh ^{-1}\left(2\left(a x+\frac{d}{x}\right)\right),
\end{gathered}
$$

or, by using the logarithms,

$$
\begin{gathered}
l_{1}=-\log \left(x^{2}\right), \\
l_{2}=-\log \left(\frac{a+d+\sqrt{(a+d)^{2}-4}}{a+d-\sqrt{(a+d)^{2}-4}}\right), \\
l_{3}=-\log \left(\frac{d+x^{2} a+\sqrt{d^{2}+2 d x^{2} a+x^{4} a^{2}-4 x^{2}}}{d+x^{2} a-\sqrt{d^{2}+2 d x^{2} a+x^{4} a^{2}-4 x^{2}}}\right),
\end{gathered}
$$

so that the jacobian

$$
J=\left(\begin{array}{lll}
\frac{\partial l_{1}}{\partial x} & \frac{\partial l_{1}}{\partial a} & \frac{\partial l_{1}}{\partial d} \\
\frac{\partial l_{2}}{\partial x} & \frac{\partial l_{2}}{\partial a} & \frac{\partial l_{2}}{\partial d} \\
\frac{\partial l_{3}}{\partial x} & \frac{\partial l_{3}}{\partial a} & \frac{\partial l_{3}}{\partial d}
\end{array}\right)
$$

becomes

$$
\left(\begin{array}{ccc}
-\frac{2}{x} & 0 & 0 \\
0 & -\frac{2}{\sqrt{a^{2}+2 a d+d^{2}-4}} & -\frac{2}{\sqrt{a^{2}+2 a d+d^{2}-4}} \\
-\frac{8 x\left(x^{2} a-d\right)}{4 x^{2} \sqrt{d^{2}+2 d x^{2} a+x^{4} a^{2}-4 x^{2}}} & -\frac{2 x^{2}}{\sqrt{d^{2}+2 d x^{2} a+x^{4} a^{2}-4 x^{2}}} & -\frac{2}{\sqrt{d^{2}+2 d x^{2} a+x^{4} a^{2}-4 x^{2}}}
\end{array}\right) .
$$

By evaluating $K=\left(J^{-1}\right)^{t}$ we can find the derivatives of $x, a$ and $d$ with respect to the $l_{*}$-coordinates. In order to evaluate the action of the vector fields $\left(\frac{\partial}{\partial l_{*}}\right)$ on 
$A, B$ and $C$ we need to calculate the matrix of the derivatives of their coefficients multiplied on the right by the inverse of the matrix itself.

In general, if $D$ is a derivative, $D(A)=\left(D\left(a_{i, j}\right)\right)_{i, j} A^{-1}$ is a 1-cocycle: in fact

$$
\begin{gathered}
D(A B)=\left(D\left(\sum a_{i, j} b_{j, k}\right)\right)_{i, j}(A B)^{-1}=\left(\sum D\left(a_{i, j}\right) b_{j, k}+a_{i, j} D\left(b_{j, k}\right)\right)_{i, j} B^{-1} A^{-1} \\
=\left(D\left(\sum a_{i, j}\right)\right)_{i, j} A^{-1}+A\left(D\left(\sum b_{j, k}\right)\right)_{i, j} B^{-1} A=D(A)+A \cdot D(B) \cdot A^{-1} .
\end{gathered}
$$

Let us notice that if we had defined $D(A)=A^{-1}\left(D\left(\sum a_{i, j}\right)\right)_{i, j}$, then the cocycle condition would have been $D(A B)=D(B)+B^{-1} D(A) B$.

The general matrix defining the corresponding 1-cocycle is then given by

$$
D(M)=\left(\begin{array}{ll}
D\left(a_{1,1}\right) a_{2,2}-D\left(a_{1,2}\right) a_{2,1} & D\left(a_{1,2}\right) a_{1,1}-D\left(a_{1,1}\right) a_{1,2} \\
D\left(a_{2,1}\right) a_{2,1}-D\left(a_{2,2}\right) a_{1,1} & D\left(a_{2,2}\right) a_{1,1}-D\left(a_{1,2}\right) a_{1,2}
\end{array}\right)
$$

where $D$ represents $\frac{\partial}{\partial l_{1}}, \frac{\partial}{\partial l_{2}}$, or $\frac{\partial}{\partial l_{3}}$. (For an explicit calculation see [Zoc95].)

3.3. The symplectic form: explicit integration. In this section we will first focus on the case $\Sigma=\Sigma(1,1)$, i.e. a torus with one boundary component. If $A$, $B, C$ are defined as in the previous section, then $\operatorname{tr}(B)=a+d$ and $\operatorname{tr}(C)=$ $\operatorname{tr}\left((A B)^{-1}\right)=\operatorname{tr}(A B)=a x+\frac{d}{x}$. In order for us to be able to glue the trinion's boundary components $B$ and $C$, we need $\operatorname{tr}(B)=\operatorname{tr}(C)$, i.e. $a+d=a x+\frac{d}{x}$, which gives the condition $d=a x$. Since we are glueing two boundary components together (in this particular case we are operating an HNN-glueing), we lose one parameter by setting the traces of the two matrices representing the boundary loops equal to each other, but we gain a new one: the Fenchel-Nielsen twist parameter $\theta$. This will be clear from the following.

The three matrices become

$$
A=\left(\begin{array}{rr}
x & 0 \\
0 & 1 / x
\end{array}\right), B=\left(\begin{array}{lr}
a & 1 \\
a^{2} x-1 & a x
\end{array}\right), C=\left(\begin{array}{ll}
a & -x \\
\frac{-a^{2} x+1}{x} & a x
\end{array}\right) .
$$

In order to find the matrix $Z$ that corresponds to the loop created by the identification of the boundary component corresponding to $B$ with the boundary component corresponding to $C$, we need to find a matrix $Z \in \mathrm{SL}(2, \mathbf{R})$ that satisfies the equality $C=Z B^{-1} Z^{-1}$.

Let $Z$ be

$$
\left(\begin{array}{ll}
u & v \\
w & z
\end{array}\right)
$$

then, by solving a non-linear system in 4 equations and 4 unknowns, i.e. $C=$ $Z B^{-1} Z^{-1}$, we get the following matrix:

$$
\left(\begin{array}{cc}
x z & v \\
-\frac{x^{2} a z-x v a^{2}+v-x a z}{x} & z
\end{array}\right)
$$

Let us note that we have not yet imposed the condition $\operatorname{det}(Z)=1$, which will give us the appropriate value of $v$, and that therefore the group of matrices (denoted by $\langle Z\rangle$ ) satisfying the above equality depends on only one parameter (here $Z$ represents a general element of the 1-parameter group satisfying the required equality).

This new parameter is related to the Fenchel-Nielsen parameter $\theta$.

More explicitly, we can solve the expression $C=Z B^{-1} Z^{-1}$ for $Z$ also by looking at the fixed points of $B$ and $C$. 

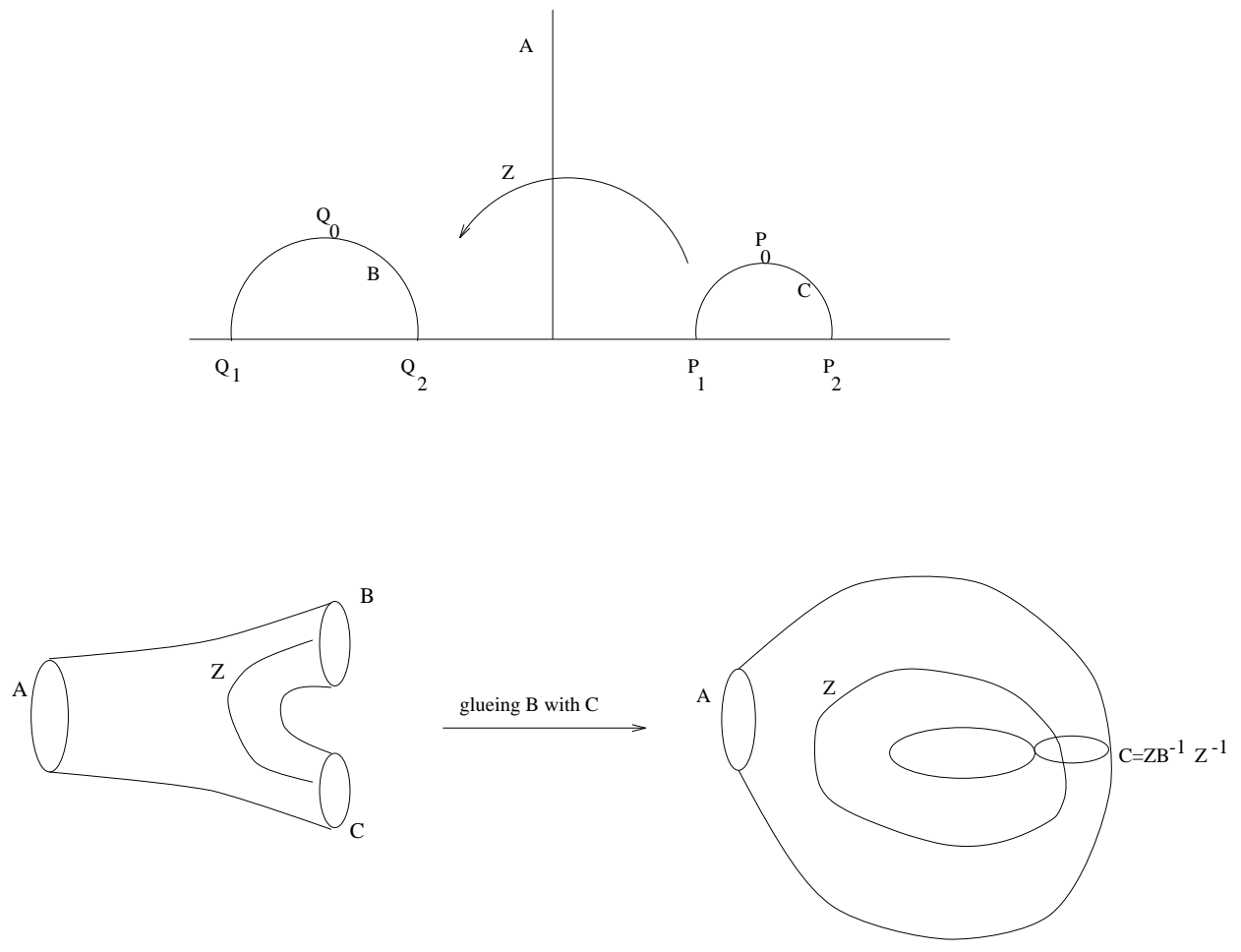

FiguRE 3. HNN-glueing

If $P$ is a fixed point for $B$, then it is a fixed point of $B^{-1}$ also, and if $Q$ is a fixed point for $C$ we need $Z$ to take $P$ to $Q$ so that we have $Q=C(Q)=$ $\left(Z B^{-1} Z^{-1}\right)(Q)=\left(Z B^{-1}\right)(P)=Z(P)=Q$.

In general $B$ and $C$ each fix a pair of points on the real axis and the geodesic connecting the pair.

In Figure 3 we denoted by $A, B$ and $C$ the geodesics fixed by the respective transformations. $Z$ will take points on the geodesic relative to $B$ to the geodesic relative to $C$. In general $Z$ will depend on a parameter $z$ that determines how far away the points on the geodesics are moved apart. The procedure to determine the Fenchel-Nielsen coordinate $\theta$ consists of choosing a point $P_{0}$ on $B$ and a point $Q_{0}$ on $C$, for example the top points of the semicircles, i.e. their median points (see Figure 3), representing the geodesics, then determine how far apart $Z$ has "hyperbolically displaced" $Z\left(P_{0}\right)$ from $Q_{0}$. The hyperbolic distance divided by the length of the geodesic represents the twist Fenchel-Nielsen coordinate $\theta$. $\theta$ measures then the "displacement angle" (see [Wol82]).

The fixed points $P_{1,2}$ for $B$ are given by

$$
P_{1,2}=\frac{a-x a \pm \sqrt{x^{2} a^{2}+2 x a^{2}+a^{2}-4}}{2 x a^{2}-2},
$$

and $Q_{1,2}$, the fixed points for $C$, by

$$
Q_{1,2}=\frac{x\left(-a+x a \pm \sqrt{x^{2} a^{2}+2 x a^{2}+a^{2}-4}\right)}{2 x a^{2}-2},
$$


and the fixed points of $A$ are given by 0 and $\infty$, making the imaginary axis the geodesic fixed by it.

By finding the parameter $\theta$ we get

$$
\theta=\frac{\left(-x z \sqrt{\Delta} \sqrt{\square}+\left(4 a^{2} x-4\right) \ln (z \sqrt{\square}+\sqrt{\Delta})\right)}{2 x(\square)}
$$

with $\Delta=\frac{z^{2} x^{3} a^{2}+2 z^{2} x^{2} a^{2}+z^{2} x a^{2}-4 x a^{2}-4 z^{2} x+4}{x}$ and $\square=x^{2} a^{2}+2 x a^{2}+a^{2}-4$.

On $\Sigma(1,1)$, the surface with fundamental group generated by $A, B, Z$ satisfying the relation $A \cdot[B, Z]=1$, the open pre-symplectic form becomes

$$
\begin{aligned}
\omega(u, v)= & -\operatorname{tr}\left(u\left(\#\left(A-A B Z B^{-1}\right)\right) \cdot v(B)\right)-\operatorname{tr}\left(u\left(\#\left(A B-A B Z B^{-1} Z^{-1}\right)\right) \cdot v(Z)\right) \\
= & -\operatorname{tr}\left(u\left((A-Z)^{-1}\right) \cdot v(B)\right)+\operatorname{tr}\left(u\left((A B)^{-1}\right) \cdot v(Z)\right) \\
= & -\operatorname{tr}\left(u\left(A^{-1}\right) \cdot v(B)\right)+\operatorname{tr}\left(u\left(Z^{-1}\right) \cdot v(B)\right) \\
& -\operatorname{tr}\left(u\left(B^{-1}\right) \cdot v(Z)\right)-\operatorname{tr}\left(B u\left(A^{-1}\right) B \cdot v(Z)\right) .
\end{aligned}
$$

We evaluate the three 1-cocycles $\left\{\frac{\partial}{\partial l_{1}}\right\},\left\{\frac{\partial}{\partial l_{2}}\right\}$ and $\left\{\frac{\partial}{\partial \theta}\right\}$ on $A, B$, and $Z$, and, by mechanically plugging in the corresponding terms in the equation representing the open pre-symplectic form (see [Zoc95]), we get the following results:

$$
\begin{gathered}
\omega\left(\frac{\partial}{\partial \theta}, \frac{\partial}{\partial l_{2}}\right)=-\omega\left(\frac{\partial}{\partial l_{2}}, \frac{\partial}{\partial \theta}\right)=-1, \\
\omega\left(\frac{\partial}{\partial \theta}, \frac{\partial}{\partial l_{1}}\right)=-\omega\left(\frac{\partial}{\partial l_{1}}, \frac{\partial}{\partial \theta}\right)=0 \\
\omega\left(\frac{\partial}{\partial l_{1}}, \frac{\partial}{\partial l_{2}}\right)=-\omega\left(\frac{\partial}{\partial l_{2}}, \frac{\partial}{\partial l_{1}}\right)=0 .
\end{gathered}
$$

Notice that we can use the glueing formula in this particular case to anticipate some of the terms of the symplectic structure by glueing two type $(1,1)$ Riemann surfaces.

In fact, since both $\frac{\partial}{\partial \theta}$ and $\frac{\partial}{\partial l_{2}}$ are zero on the loop $A$, there is no propagation, i.e. the vector fields relative to the coordinates on one of the type $(1,1)$ surfaces are zero on the other. The other essential hypothesis we will need is that $A$ be diagonal.

When we glue the two surfaces together we conjugate the fundamental group of the surface to the "right" by a matrix

$$
T=\left[\begin{array}{cc}
0 & t \\
-1 / t & 0
\end{array}\right]
$$

Then the new fundamental group for the right surface is generated by the matrices $T A T^{-1}, T B T^{-1}, T C T^{-1}$ (see [Wol82]).

The 1-cocycle $u$, evaluated on the matrix $T M T^{-1}$ has value

$$
\begin{gathered}
u\left(T M T^{-1}\right)=u(T)+T u\left(M T^{-1}\right) T^{-1} \\
=u(T)+T u(M) T^{-1}-T M T u(T) M^{-1} T^{-1} .
\end{gathered}
$$

Since the vector fields relative to the loops not corresponding to the boundary component $A$ are zero on $T$, and because we have no propagation, the evaluation 
of the symplectic form on those vector fields reduces to the symplectic form on one of the two distinct Riemann surfaces.

Of course, if $\frac{\partial}{\partial l}$ or $\frac{\partial}{\partial \theta}$ are the Fenchel-Nielsen coordinates for $A$, then neither the fact that $\frac{\partial}{\partial l}(T)=0$ or $\frac{\partial}{\partial \theta}(T)=0$, nor the hypothesis that there is no propagation, is true anymore.

For the Fenchel-Nielsen coordinates relative to the other loops we could satisfy those hypotheses by choosing our representation so that the boundary loops to be glued have a diagonal representation. We can generalise the process to evaluate the symplectic form for any Riemann surface of genus $g \geq 2$.

\section{Generalising to projective structures: An introduction to PGL $(3, \mathbf{R})$.}

The definition of a convex real projective structure on a Riemann surface generalises the definition of hyperbolic structure.

Whereas the universal covering space for a Riemann surface endowed with a hyperbolic structure is the hyperbolic space (the case genus $\geq 2$ ), for a Riemann surface endowed with a real projective structure, the universal covering space is the projective plane $\mathbf{R} P^{2}$ (it should be noted that we will use the term "Riemann surface" to denote an oriented differentiable two dimensional manifold, closed or not, and not a surface endowed with a complex structure as is customary). Moreover, while the group of Moebius transformations, $\operatorname{PSL}(2, \mathbf{R})$, acts in the hyperbolic structure case, for projective structures the group considered is $\operatorname{PGL}(3, \mathbf{R})$, the group of projective transformations.

A [convex] real projective manifold (Riemann surface) is then a quotient $M=$ $\Omega / \Gamma$, where $\Omega \in \mathbf{R} P^{2}$ is a [convex] domain and $\Gamma \in \operatorname{PGL}(3, \mathbf{R})$ is a discrete group of projective transformations acting properly on $M$.

We will now introduce all the basic definitions and facts about convex real projective structures that we need in order to symplectically integrate the symplectic form defined on $\operatorname{Hom}\left(\pi_{1}(\Sigma), \operatorname{PGL}(3, \mathbf{R})\right) / \operatorname{PGL}(3, \mathbf{R})$.

\subsection{Projective manifolds.}

Definition 4.1.1. PGL $(3, \mathbf{R})$ represents the group of projective transformations.

A linear map from $\mathbf{R}^{3}$ to $\mathbf{R}^{3}$ is an element of $\mathrm{GL}(3, \mathbf{R})$ and it leaves invariant the lines through the origin in $\mathbf{R}^{3}$. As such it induces a transformation from $\mathbf{R} P^{2}$ to $\mathbf{R} P^{2}$. Of course the group $\mathbf{R}^{*}$ of scalar matrices acts trivially on $\mathbf{R} P^{2}$; therefore the quotient $\operatorname{PGL}(3, \mathbf{R})=\mathrm{GL}(3, \mathbf{R}) / \mathbf{R}^{*}$ acts on $\mathbf{R} P^{2}$, and we call it the group of projective transformations.

We note that since PGL $(3, \mathbf{R})$ is a group of lateral classes, we can always choose a representative matrix of determinant 1 that defines an element in $\operatorname{SL}(3, \mathbf{R})$. Viceversa, an element in $\operatorname{SL}(3, \mathbf{R})$ defines a unique class in $\operatorname{PGL}(3, \mathbf{R})$. For that reason we may identify the group of projective transformations $\operatorname{PGL}(3, \mathbf{R})$ with $\operatorname{SL}(3, \mathbf{R})$.

Definition 4.1.2. Let $\Omega \in \mathbf{R P}^{2}$ be an open connected set. We define a map $\phi: \Omega \rightarrow \mathbf{R} P^{2}$ to be a locally projective transformation if there exists a projective transformation $\Phi$ that when restricted to $\Omega$ coincides with $\phi$. If $\Omega$ is not connected, $\phi$ is locally projective if its restrictions to each connected component of $\Omega$ are all locally projective transformations. 
Definition 4.1.3. A Riemann surface $\Sigma$ is endowed with a projective structure if:

i) There exists a covering $\mathcal{U}$ of $\Sigma$ such that each $U \in \mathcal{U}$ is diffeomorphic to an open set in $\mathbf{R} P^{2}$.

ii) For every $U$ and $V$ in $\mathcal{U}$ such that $U \cap V \neq \emptyset$, the transition functions that provide the change of coordinates are locally projective transformations.

Notice the correspondence with the hyperbolic case; in that case the Riemann surface has to possess a covering of open sets holomorphically equivalent to open sets in $\mathbf{C}$, i.e. diffeomorphic to open sets in $\mathbf{C}$ and such that the transition functions are holomorphic ([Wel80]). Similarly, we may require the open sets to be homeomorphic to the hyperbolic upper plane and the transition functions to be Moebius transformations (hyperbolic transformations).

The analogy is therefore clear: for real projective structures, $\mathbf{R} P^{2}$ plays the role that $\mathbf{H}$, the hyperbolic upper half-plane, plays for hyperbolic structures, and the group of projective transformations replaces the group of Moebius transformations.

Continuing this analogy, it is then clear that in the $\mathbf{R} P^{2}$ case we regard the fundamental group of the Riemann surface as having a representation into a subgroup of PGL $(3, \mathbf{R})$ instead of $\mathrm{SL}(2, \mathbf{R})$, and that a Riemann surface endowed with a real projective structure is an element in $\operatorname{Hom}\left(\pi_{1}(\Sigma), \operatorname{PGL}(3, \mathbf{R})\right)$, while a Riemann surface with a hyperbolic structure is an element of $\operatorname{Hom}\left(\pi_{1}(\Sigma), \operatorname{PSL}(2, \mathbf{R})\right)$.

4.2. Convex $\mathbf{R} P^{2}$-structures. Projective structures are defined by [classes of] representations from $\pi_{1}(M)$ into $\mathrm{PGL}(3, \mathbf{R})$. In particular $M$ is isomorphic to a quotient $\Omega / \Gamma$, where $\Omega \subset \mathbf{R} P^{2}$ and $\Gamma \subset \mathrm{SL}(3, \mathbf{R})$. We will focus on convex real projective structures, which means we add the further requirement that $\Omega$ be convex in $\mathbf{R} P^{2}$.

Theorem 4.2.1. Suppose $\chi(M)<0$, and $M=\Omega / \Gamma$ a compact convex real projective manifold. Then:

i) $\Omega$ is strictly convex.

ii) Either $\partial \Omega$ is a conic, or it is $C^{1}$ but not $C^{2-t}$ for some $t>0$.

iii) Any $\gamma \in \Gamma$ has positive eigenvalues.

This theorem explains why we are mainly interested in convex projective structures. In fact, whenever $\partial \Omega$ is a conic, the projective structure reduces to a hyperbolic structure. Convex projective structures are then clearly a generalisation of hyperbolic structures.

The Klein model, in fact, offers a geometric way to embed into $\mathbf{R} P^{2}$ the hyperbolic plane, whose boundary is a conic. A choice of a conic in $\mathbf{R} P^{2}$ is then the geometric equivalent to choosing an algebraic embedding of $\operatorname{PSL}(2, \mathbf{R})$ into $\operatorname{PGL}(3, \mathbf{R})$.

In [Hit92], Hitchin proves the existence of a connected component of the spaces of representations $\operatorname{Hom}\left(\pi_{1}(\Sigma), \operatorname{PSL}(n, \mathbf{R})\right) / \operatorname{PSL}(n, \mathbf{R})$, a component that he names

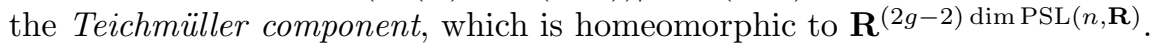

For $\operatorname{PSL}(2, \mathbf{R})$ this connected component is Teichmüller space; in the general case it contains Teichmüller space in the sense that there exists a distinguished 3 -dimensional subgroup of $\operatorname{PSL}(n, \mathbf{R})$ isomorphic to $\operatorname{PSL}(2, \mathbf{R})$, which embeds the representations of $\pi_{1}(\Sigma)$ into a subspace, homeomorphic to Teichmüller space, of the Teichmüller component. Goldman and Choi ([CG93]) proved that the space of convex real projective structures is the Teichmüller component defined by Hitchin. 
Note that we can algebraically write the embedding

$$
\operatorname{Hom}\left(\pi_{1}(\Sigma), \operatorname{PSL}(2, \mathbf{R})\right) \subset \operatorname{Hom}\left(\pi_{1}(\Sigma), \operatorname{PGL}(3, \mathbf{R})\right)
$$

by identifying $\operatorname{PSL}(2, \mathbf{R})$ with a connected component of $\mathrm{SO}(2,1) \subset \mathrm{GL}(3, \mathbf{R})$.

Let

$$
A=\left(\begin{array}{ll}
a & b \\
c & d
\end{array}\right) \in \mathrm{SL}(2, \mathbf{R})
$$

and

$$
X=\left(\begin{array}{cc}
x & y \\
z & -x
\end{array}\right) \in \mathcal{S} \mathcal{L}(2, \mathbf{R}) .
$$

The norm $\|X\|^{2}=\operatorname{tr}(X \cdot X)=2 x^{2}+2 y z$ is given in $\mathbf{R}^{3}$ by the inner product associated to the matrix

$$
J=\left(\begin{array}{lll}
2 & 0 & 0 \\
0 & 0 & 1 \\
0 & 1 & 0
\end{array}\right)
$$

The map

$$
A d_{A}(X)=A \cdot X \cdot A^{-1}=\left(\begin{array}{cc}
(a d+b c) x-a c y+b d z & -2 a b x+a^{2} y-b^{2} z \\
2 c d x-c^{2} y+d^{2} z & -(a d+b c) x+a c y-b d z
\end{array}\right)
$$

is given in $\mathbf{R}^{3}$ by the matrix

$$
P=\left(\begin{array}{ccc}
a d+b c & -2 a b & 2 c d \\
-a c & a^{2} & -c^{2} \\
b d & -b^{2} & d^{2}
\end{array}\right)
$$

The map

$$
\begin{array}{ccc}
\mathrm{SL}(2, \mathbf{R}) & \rightarrow & \mathrm{GL}(3, \mathbf{R}), \\
A & \mapsto & P,
\end{array}
$$

is a monomorphism of groups.

Moreover, since it can easily be seen that there exists a matrix $Q$ such that

$$
Q \cdot J \cdot Q^{t}=I_{-}=\left(\begin{array}{ccc}
1 & 0 & 0 \\
0 & -1 & 0 \\
0 & 0 & 1
\end{array}\right)
$$

with

$$
Q=\left(\begin{array}{ccc}
1 / 2 & 0 & 0 \\
0 & 1 / \sqrt{2} & -1 / \sqrt{2} \\
0 & 1 / \sqrt{2} & 1 / \sqrt{2}
\end{array}\right),
$$

and since clearly $P \cdot J \cdot P^{t}=J$, we have that $\left(Q P Q^{-1}\right) I_{-}\left(Q P Q^{-1}\right)^{t}=I_{-}$, i.e. $Q P Q^{-1} \in \mathrm{SO}(2,1)$.

The map

$$
\begin{array}{cc}
\mathrm{SL}(2, \mathbf{R}) & \rightarrow \mathrm{SO}(2,1), \\
A & \mapsto Q P Q^{-1},
\end{array}
$$

is then a well defined isomorphism, i.e. $\operatorname{PSL}(2, \mathbf{R})$ is isomorphic with a connected component of $\mathrm{SO}(2,1)$. 
4.3. The $G$-coordinates. The purpose of this section is to generalise some of the theory of hyperbolic structures to projective structures. In particular, we will generalise the Fenchel-Nielsen coordinates and the definition of a twist field (we will define a generalised twist field) to give global coordinates on the new space.

On a trinion, we will see that we need two parameters for each boundary loop (analogous to the "trace") plus two "inner" coordinates, and that the glueing coordinates include an "extension" parameter $\beta$ together with the "twist" parameter $\theta$ defined in the previous chapter. Many of the definitions and theorems given here are due to Goldman [Gol84], [Gol86] and [Gol90].

First of all we will define new global coordinates on this space by generalising the approach used in the previous chapter. We start by considering a trinion $\Sigma(0,3)$. Once we fix a representation $\phi$, the elements relative to the boundary components can be regarded as conjugacy classes of matrices in $\operatorname{PGL}(3, \mathbf{R})$. As such they have three eigenvalues $\lambda, \mu$ and $\nu$ satisfying the relation $\lambda \mu \nu=1$, with $0<\lambda \leq \mu \leq \nu$. Therefore two of those eigenvalues completely determine the conjugacy class of the matrix.

In order to define new coordinates that extend the coordinates given in $\operatorname{PSL}(2, \mathbf{R})$, we need to define functions of those eigenvalues that, when restricted to $\operatorname{PSL}(2, \mathbf{R})$, produce the Fenchel-Nielsen coordinates.

For a matrix $M \in$ image of $(\operatorname{PSL}(2, \mathbf{R})) \subset \operatorname{PGL}(3, \mathbf{R})$, one of the eigenvalues has to be 1 and the other two have to be reciprocals of one another. We remind the reader that the Fenchel-Nielsen coordinate $l$, the length, was defined by $l=2 \cosh ^{-1}\left(\left|\frac{\operatorname{tr}(M)}{2}\right|\right)$. The expression $\cosh ^{-1}(t)$ can be rewritten as $\cosh ^{-1}(t)=$ $\log \left(t+\sqrt{\left(t^{2}-1\right)}\right)$; therefore we can rewrite $l=2 \log (\nu)=\log \left(\nu^{2}\right)$ by substituting $\operatorname{tr}(M)$ for $t$, with $\operatorname{tr}(M)=\nu+1 / \nu$. If we define a new coordinate $l=\log \left(\frac{\nu}{\lambda}\right)$, we then have that the "new" coordinate $l$ extends the "old" coordinate $l$, and they agree for $\operatorname{PSL}(2, \mathbf{R})$. We can define another coordinate $m=\frac{3}{2} \log (\mu)$ that is also invariant under conjugation.

4.4. A geometric model for $P G L(3, \mathbf{R})$-structures. Let $M$ be given by

$$
M=\left(\begin{array}{ccc}
\lambda & 0 & 0 \\
0 & \mu & 0 \\
0 & 0 & \nu
\end{array}\right)
$$

then, for the action on $\mathbf{R} P^{2}$, we define

Definition 4.4.1. Fix $(M) \stackrel{\text { def }}{=}$ repelling fixed point = fixed point corresponding to the smallest eigenvalue $\lambda$,

$\operatorname{Fix}_{0}(M) \stackrel{\text { def }}{=}$ saddle fixed point $=$ fixed point corresponding to the middle eigenvalue $\mu$,

Fix $_{+}(M) \stackrel{\text { def }}{=}$ attractive fixed point $=$ fixed point corresponding to the largest eigenvalue $\nu$.

The line connecting the attractive and repelling points will be referred to as the principal line $l(M)$. Sometimes we will also refer to the repelling, saddle and attractive points of $M$ as $r(M), s(M)$ and $a(M)$ respectively. In the particular case when $M$ is diagonal and $\gamma>\mu>\nu>0$ we have: $a(M)=[1,0,0], s(M)=[0,1,0]$, and $r(M)=[0,0,1]$. 


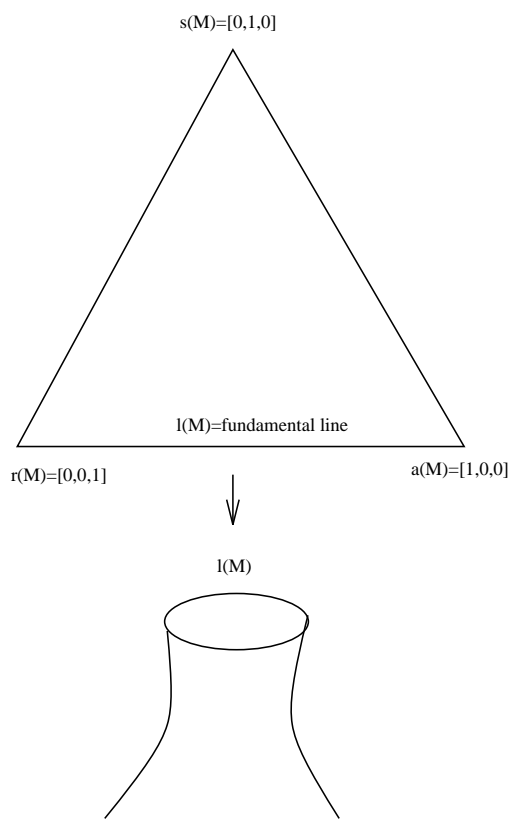

Figure 4. Principal line for a diagonal projective transformation.

The principal line of a matrix $M$ relative to a boundary component, i.e. the image $\phi(\gamma)$ for a representation $\phi$ of the loop $\gamma$ relative to the boundary loop, represents the lift of the same boundary component (see Figure 4).

In projective geometry we can define a metric, the Hilbert metric, that allows us to measure the distance between two points and their "displacement" under the action of a matrix in $\mathrm{PGL}(3, \mathbf{R})$.

Definition 4.4.2. The cross ratio of four points $\left(z_{1}, z_{2}, z_{3}, z_{4}\right)$ on a projective line is

$$
C R=\frac{\left(z_{1}-z_{3}\right)\left(z_{2}-z_{4}\right)}{\left(z_{1}-z_{4}\right)\left(z_{2}-z_{3}\right)}
$$

When two of these terms are $\infty$ we assume that their quotient is 1 .

It is not difficult to prove that the cross ratio $C R$ is independent of the choice of coordinates; it then represents a projective invariant for any 4 collinear points in projective space.

By using the definition of cross ratio, we can now define the Hilbert distance for pairs of points $(a, b)$ situated on a segment $\overline{x y}$.

Definition 4.4.3. The Hilbert distance $h(a, b)$ between two points $a$ and $b$ lying on the oriented segment $\overrightarrow{x y}$, where $a$ is the first point, is given by

$$
h(a, b)=\log C R(a, b, x, y) .
$$

The Hilbert distance defines a metric on convex subsets $\Omega \subset \mathbf{R} P^{2}$ that is called the Hilbert metric ([BK53], [Kob82], [Kob84]).

If we measure the Hilbert distance between two points $a$ and $b=M(a)$ on the principal line we find that $a=[\alpha, 0, \gamma], M(a)=[\lambda \alpha, 0, \nu \gamma]$, and $h(a, M(a))=\log \left(\frac{\nu}{\lambda}\right)$; 


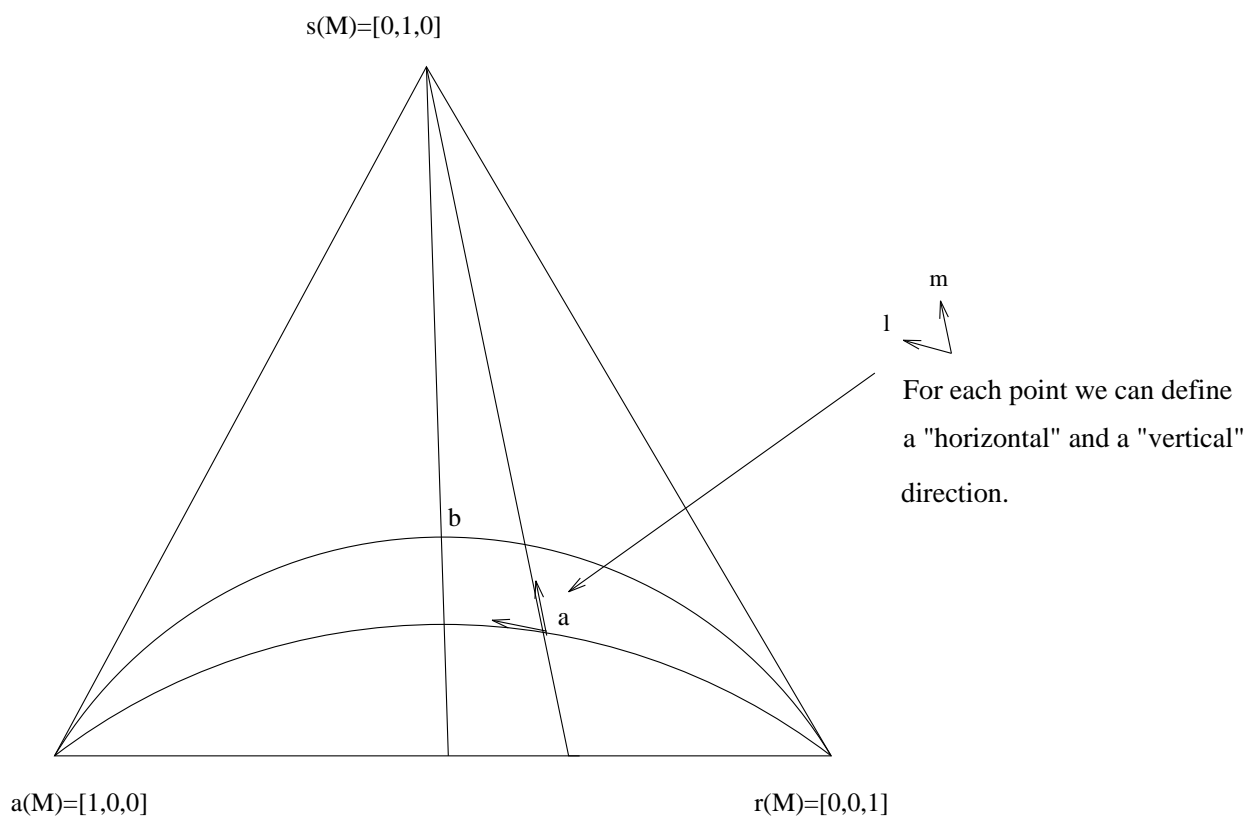

FiguRE 5. Geometrical interpretation of the coordinates $l$ and $m$

$\log \left(\frac{\nu}{\lambda}\right)$ then represents the displacement induced by a matrix on a point lying on the principal line (i.e. the boundary loop).

Then, the coordinate $l$ measures the distance between the points $a$ and $M(a)$. If $M \in \operatorname{PSL}(2, \mathbf{R})$, then $l$ equals the hyperbolic distance between the two points, and in general if we consider convex real projective structures, i.e. the boundary of the open subset $\Omega \subset \mathbf{R} P^{2}$ is a conic, then the Hilbert metric is the hyperbolic metric.

The Hilbert metric then represents the correct generalisation of the hyperbolic metric for $\operatorname{PGL}(3, \mathbf{R})$-structures.

However, let us choose a point $a$ not on the principal line.

If $M \in \operatorname{PSL}(2, \mathbf{R})$, then the orbit of the point will represent the "horizontal" direction which is given by $\left[\frac{1}{\nu} \alpha, \beta, \nu \gamma\right]$. In other words, $\operatorname{PSL}(2, \mathbf{R})$ moves points in $\mathbf{R} P^{2}$ from the repelling to the attractive point "horizontally" (see Figure 5).

The Lie algebra of the group of the diagonal matrices of $\operatorname{PSL}(2, \mathbf{R})$ in $\operatorname{SL}(3, \mathbf{R})$ is given by

$$
\left\{\left(\begin{array}{lll}
t & 0 & 0 \\
0 & 0 & 0 \\
0 & 0 & -t
\end{array}\right)\right\} \subset\left\{\left(\begin{array}{lll}
t+s & 0 & 0 \\
0 & -2 s & 0 \\
0 & 0 & -t+s
\end{array}\right)\right\}
$$

so that the group

$$
H=\left\{\left(\begin{array}{lll}
\frac{1}{\sqrt{\mu}} & 0 & 0 \\
0 & \mu & 0 \\
0 & 0 & \frac{1}{\sqrt{\mu}}
\end{array}\right)\right\}
$$


corresponding to the Lie algebra generated by

$$
\left\{\left(\begin{array}{ccc}
s & 0 & 0 \\
0 & -2 s & 0 \\
0 & 0 & s
\end{array}\right)\right\}
$$

is "orthogonal" to $\operatorname{PSL}(2, \mathbf{R})$ in $\operatorname{SL}(3, \mathbf{R})$.

$H$ moves a point $a$ in $\mathbf{R} P^{2}$ along the line through $a$ connecting $[0,1,0]$ with the principal line. That represents the "vertical" direction (see Figure 5).

In other words, for subgroups of diagonal matrices, the PSL(2,R)-orbit will be called "horizontal", and the pencil based at $[0,1,0]$ will give the "vertical" direction.

The image $b$ of $a=(\alpha, \beta, \gamma)$ by $\mathrm{H}$ is given by

$$
\left[\frac{1}{\sqrt{\mu}} \alpha, \mu \beta, \frac{1}{\sqrt{\mu}} \gamma\right]
$$

so the Hilbert distance

$$
\begin{aligned}
h(a, b) & =\log \left\{C R\left([\alpha, \beta, \gamma],\left[\frac{1}{\sqrt{\mu}} \alpha, \mu \beta, \frac{1}{\sqrt{\mu}} \gamma\right],[0,1,0],\left[\frac{\alpha}{1-\beta}, 0, \frac{\gamma}{1-\beta}\right]\right)\right\} \\
& =\log \left\{C R\left([\alpha, \beta, \gamma],\left[\alpha, \sqrt{\mu^{3}} \beta, \gamma\right],[0,1,0],[\alpha, 0, \gamma]\right)\right\} \\
& =\log \left\{\sqrt{\mu^{3}}\right\}=\frac{3}{2} \log (\mu) .
\end{aligned}
$$

The vertical line passing through the point $[\alpha, \beta, \gamma]$ is then given by $[\alpha, t \beta, \gamma]$.

The line representing the horizontal direction is given by the tangent line at $[\alpha, \beta, \gamma]$ of the orbit $\left[\alpha, t \beta, t^{2} \beta\right]$ through the point, and is defined by $\left[\frac{2}{t+1} \alpha, \beta, \frac{2 t}{t+1} \gamma\right]$.

Therefore

$$
M=\left(\begin{array}{ccc}
\lambda & 0 & 0 \\
0 & \mu & 0 \\
0 & 0 & \nu
\end{array}\right) \in \operatorname{SL}(3, \mathbf{R})
$$

moves a point $a$ "horizontally" by $\log \left(\frac{\nu}{\gamma}\right)$ and "vertically" by $\frac{3}{2} \log (\mu)$ (see Figure 5$)$.

Definition 4.4.4. The quantity $\log \left(\frac{\nu}{\gamma}\right)$ represents the (horizontal) translation by the $\operatorname{PSL}(2, \mathbf{R})$-component of $\operatorname{SL}(3, \mathbf{R})$, and $\frac{3}{2} \log (\mu)$ represents its "orthogonal" translation.

The corresponding coordinates $l=\log \left(\frac{\nu}{\gamma}\right)$ and $m=\frac{3}{2} \log (\mu)$ for each boundary component are part of the $G$-coordinates for projective structures on a trinion.

There are two more coordinates internal to the trinion.

On a Riemann surface other than a trinion there are also other coordinates generalising the Fenchel-Nielsen twist coordinate $\theta$, which are denoted by $\theta$ and $\beta$. These are also well-defined functions on a Riemann surface; in fact, for each pair of pants we can select three pairs of points $\left(p_{i}, q_{i}\right)$, using the cross-ratio and intersections of lines (see Figure 6).

The measure of how far apart these two points are on the corresponding boundary component of two glued trinions gives the functions $\theta$ and $\beta$. (The vector fields of the coordinates $\theta$ and $\beta$ here defined correspond to "twists" generalised to the PGL $(3, \mathbf{R})$ case. For a precise and complete discussion of generalised twistfields see $[\mathrm{Gol} 86]$.) A heuristic count for $\operatorname{dim}\left(\operatorname{Hom}\left(\pi_{1}(\Sigma), \operatorname{SL}(3, \mathbf{R})\right) / \operatorname{SL}(3, \mathbf{R})\right)$ gives $16(g-1)+8 n$. 

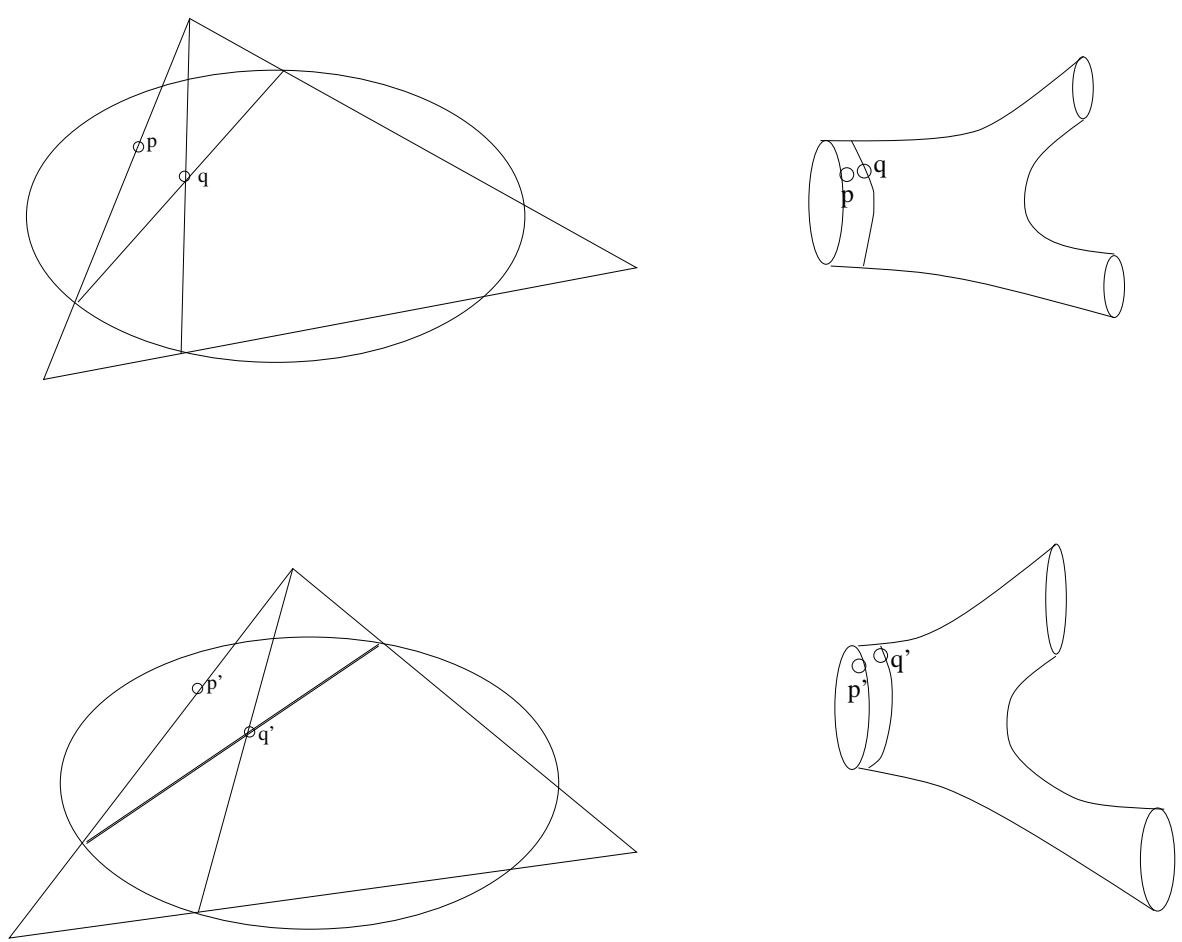

FIGURE 6. Choice of "base points" to determine the coordinates $\theta$ and $\beta$

In fact $\pi_{1}(\Sigma)$ has $2 g+n-1$ generators, while $\mathrm{SL}(3, \mathbf{R})$ has dimension 8 , so that

$$
\operatorname{dim}\left(\operatorname{Hom}\left(\pi_{1}(\Sigma), \operatorname{SL}(3, \mathbf{R})\right)\right)=8(2 g+n-1) .
$$

By reducing to the quotient $\operatorname{Hom}\left(\pi_{1}(\Sigma), \mathrm{SL}(3, \mathbf{R})\right) / \mathrm{SL}(3, \mathbf{R})$ we decrease the count by 8 , getting the final number $16(g-1)+8 n$.

For a trinion, that would correspond to dimension $8(g=0, n=3)$.

If we count two coordinates, $l$ and $m$, for each boundary component, that would total 6 coordinates, which means that in order to describe the space of projective structures on a trinion we need two further "inner" coordinates that we denote by $s$ and $t$.

The set $\left(l_{1}, m_{1}, l_{2}, m_{2}, l_{3}, m_{3}, s, t\right)$ provides the whole set of coordinates needed to describe the space of projective structures on a trinion (see [Gol90]). Goldman (also in [Gol90]) provides a way to describe the three matrices $A, B, C$ satisfying the relation $C B A=1$, relative to the boundary components of a trinion, for the space of representations of the fundamental group of a surface into PGL $(3, \mathbf{R})$. He proves that the space of representations of the fundamental group of a Riemann surface into $\mathrm{PGL}(3, \mathbf{R})$ is equivalent to a certain geometric construction satisfying determined properties.

That geometric construction is fully determined by two of the three vertices $u, v, w$ (see Figure 7) and by the eigenvalues of the three matrices $A, B, C$ mapping one triangular region over another. In fact, one of the three vertices can be chosen arbitrarily because of the group action by conjugation. We choose $w=\left(u_{3}, v_{3}, 1\right)=$ $(2,2,1)$. Therefore the two vertices $u$ and $v$ are determined by four coordinates, and 


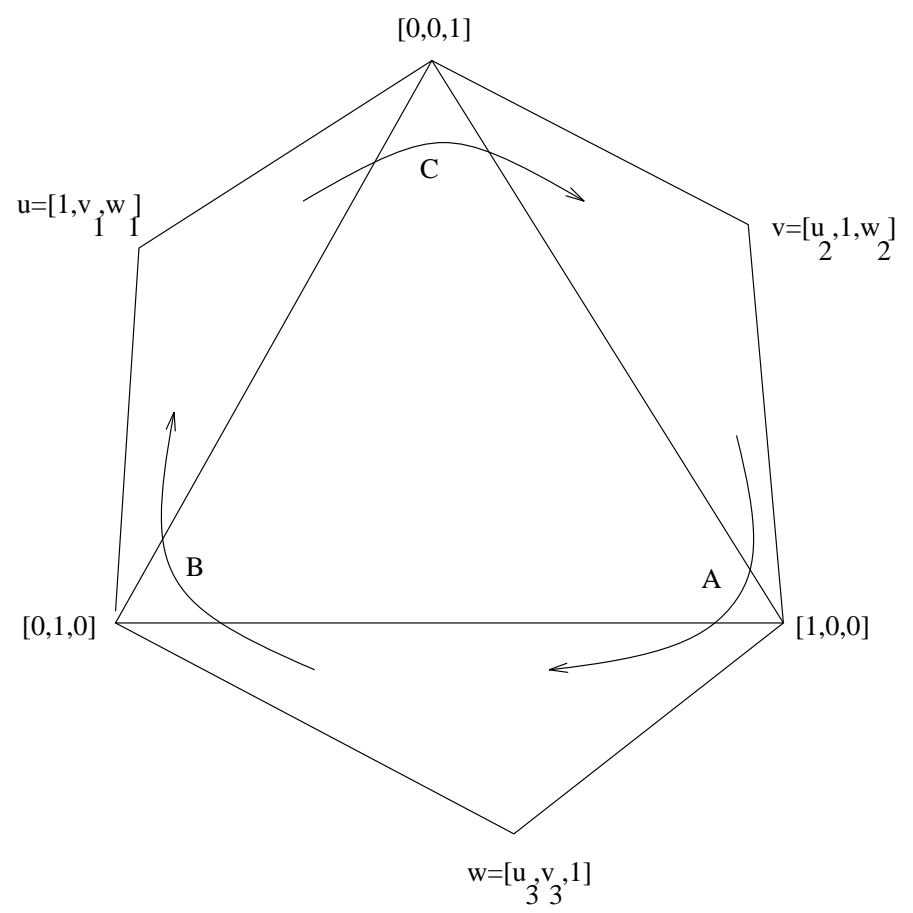

FiguRe 7. Geometric description of the moduli space of $\mathbf{R} P^{2}$ structures

the eigenvalues by four more parameters. A priori we have 9 eigenvalues; however, because of the relation $C B A=1$ and because $\operatorname{det} A=\operatorname{det} B=\operatorname{det} C=1$, the independent eigenvalues are only four. That, as expected, gives 8 independent coordinates. Goldman writes down explicitly the three matrices $A, B, C$ in terms of their eigenvalues and the coordinates of the points $u, v$ and $w$.

4.5. A geometrical description. As Goldman proves in his paper ([Gol90]) using a direct and constructive approach, the matrices $\Phi(A), \Phi(B), \Phi(C)$ relative to the boundary loops $A, B, C$ are as follows (again we will write $A, B, C$ for $\Phi(A), \Phi(B), \Phi(C)$ whenever there should be no confusion whether we are referring to the loops or their image, under the representation $\Phi$, into $\operatorname{PSL}(3, \mathbf{R}))$ :

$$
\begin{aligned}
A & =\left(\begin{array}{ccc}
\alpha_{1} & \alpha_{1} u_{2}+\gamma_{1} u_{3} w_{2} & \gamma_{1} u_{3} \\
0 & -\beta_{1}+\gamma_{1} v_{3} w_{2} & \gamma_{1} v_{3} \\
0 & -\gamma_{1} w_{2} & -\gamma_{1}
\end{array}\right), \\
B & =\left(\begin{array}{ccc}
-\alpha_{2} & 0 & -\alpha_{2} u_{3} \\
\alpha_{2} v_{1} & \beta_{2} & \beta_{2} v_{3}+\alpha_{2} u_{3} v_{1} \\
\alpha_{2} w_{1} & 0 & -\gamma_{2}+\alpha_{2} u_{3} w_{1}
\end{array}\right), \\
C & =\left(\begin{array}{lll}
-\alpha_{3}+\beta_{3} u_{2} v_{1} & \beta_{3} u_{2} & 0 \\
-\beta_{3} v_{1} & -\beta_{3} & 0 \\
\gamma_{3} w_{1}+\beta_{3} w_{2} v_{1} & \beta_{3} w_{2} & -\gamma_{3}
\end{array}\right) .
\end{aligned}
$$


The advantage of writing $A, B, C$ in this fashion is clear: in fact $\alpha_{1}, \beta_{2}, \gamma_{3}$ are immediately identified as eigenvalues, and the other two eigenvalues can be easily found as the two eigenvalues of the remaining 2 by 2 minor.

Without loss of generality we can assume that $\mu_{1}=\alpha_{1}, \mu_{2}=\beta_{2}, \mu_{3}=\gamma_{3}$, i.e. that, because of the choice of the representation, the three eigenvalues $\alpha_{1}, \beta_{2}$ and $\gamma_{3}$ are the "middle" eigenvalues. It is clear from the group condition that the three matrices satisfy, i.e. $C B A=1$ and $\operatorname{det}(A)=\operatorname{det}(B)=\operatorname{det}(C)=1$, that $\alpha_{1} \alpha_{2} \alpha_{3}=\beta_{1} \beta_{2} \beta_{3}=\gamma_{1} \gamma_{2} \gamma_{3}=\alpha_{1} \beta_{1} \gamma_{1}=\alpha_{2} \beta_{2} \gamma_{2}=\alpha_{3} \beta_{3} \gamma_{3}=1$. Again, as in the previous section, $u_{3}=v_{3}=2$.

Also, for reasons that will soon be clear, we rename the variables $v_{1}$ and $u_{2}, s$ and $t$ respectively. In this way it will be easier to reduce this case to the $\operatorname{PSL}(2, \mathbf{R})$ case where $\alpha_{1}=\beta_{2}=\gamma_{3}=1$. By finding the other eigenvalues, we obtain the following global coordinates:

$$
\begin{aligned}
& l_{1}=\left|\ln \left(\frac{-\alpha_{1} \beta_{1}^{2}+2 w_{2}-1+\sqrt{\alpha_{1}^{2} \beta_{1}^{4}-4 \alpha_{1} \beta_{1}^{2} w_{2}-2 \alpha_{1} \beta_{1}^{2}+4 w_{2}^{2}-4 w_{2}+1}}{-\alpha_{1} \beta_{1}^{2}+2 w_{2}-1-\sqrt{\alpha_{1}^{2} \beta_{1}^{4}-4 \alpha_{1} \beta_{1}^{2} w_{2}-2 \alpha_{1} \beta_{1}^{2}+4 w_{2}^{2}-4 w_{2}+1}}\right)\right|, \\
& m_{1}=\frac{3}{2} \log \left(\alpha_{1}\right), \\
& l_{2}= \\
& \left|\ln \left(\frac{\alpha_{2}^{2} \beta_{2}-2 \alpha_{2}{ }^{2} w_{1} \beta_{2}+1+\sqrt{\alpha_{2}{ }^{4} \beta_{2}{ }^{2}-4 \alpha_{2}{ }^{4} \beta_{2}{ }^{2} w_{1}-2 \alpha_{2}{ }^{2} \beta_{2}+4 \alpha_{2}{ }^{4} w_{1}{ }^{2} \beta_{2}{ }^{2}-4 \alpha_{2}{ }^{2} w_{1} \beta_{2}+1}}{\alpha_{2}{ }^{2} \beta_{2}-2 \alpha_{2}{ }^{2} w_{1} \beta_{2}+1-\sqrt{\alpha_{2}{ }^{4} \beta_{2}{ }^{2}-4 \alpha_{2}{ }^{4} \beta_{2}{ }^{2} w_{1}-2 \alpha_{2}{ }^{2} \beta_{2}+4 \alpha_{2}{ }^{4} w_{1}^{2} \beta_{2}{ }^{2}-4 \alpha_{2}{ }^{2} w_{1} \beta_{2}+1}}\right)\right|, \\
& m_{2}=\frac{3}{2} \log \left(\beta_{2}\right) \\
& l_{3}= \\
& \left|\ln \left(\frac{-s \alpha_{1} t \alpha_{2}+\beta_{2} \beta_{1}+\alpha_{1} \alpha_{2}+\sqrt{s^{2} \alpha_{1}^{2} t^{2} \alpha_{2}^{2}-2 s \alpha_{1} t \alpha_{2} \beta_{2} \beta_{1}-2 s \alpha_{1}^{2} t \alpha_{2}^{2}+\left(\alpha_{1} \alpha_{2}-\beta_{1} \beta_{2}\right)^{2}}}{-s \alpha_{1} t \alpha_{2}+\beta_{2} \beta_{1}+\alpha_{1} \alpha_{2}-\sqrt{s^{2} \alpha_{1}^{2} t^{2} \alpha_{2}^{2}-2 s \alpha_{1} t \alpha_{2} \beta_{2} \beta_{1}-2 s \alpha_{1}{ }^{2} t \alpha_{2}{ }^{2}+\left(\alpha_{1} \alpha_{2}-\beta_{1} \beta_{2}\right)^{2}}}\right)\right|, \\
& m_{3}=\frac{3}{2} \log \left(\gamma_{3}\right) \text {. }
\end{aligned}
$$

These coordinates are a direct generalisation of the Fenchel-Nielsen coordinates as explained in chapter 1 .

Since projective structures are a generalisation of hyperbolic structures, we can recover the Fenchel-Nielsen coordinates by simply imposing some conditions on the entries of the matrices $A, B$, and $C$.

First of all, as we already mentioned, $\alpha_{1}=\beta_{2}=\gamma_{3}=1$ gives $m_{1}=m_{2}=m_{3}=0$. Also, since $\alpha_{1} \alpha_{2} \alpha_{3}=\beta_{1} \beta_{2} \beta_{3}=\gamma_{1} \gamma_{2} \gamma_{3}=\alpha_{1} \beta_{1} \gamma_{1}=\alpha_{2} \beta_{2} \gamma_{2}=\alpha_{3} \beta_{3} \gamma_{3}=1$, that implies $\alpha_{1}=\beta_{2}$ and $\beta_{1}=1 / \alpha_{2}$. Moreover, if the projective structure actually is a hyperbolic structure, then the boundary $\partial \Omega$ of the domain $\Omega \subset \mathbf{R} P^{2}$ is a conic. That sets conditions on the vertices $u, v$ and $w$, and consequently on $w_{1}, w_{2}, s$ and $t$. More precisely, the condition that all those points lie on a conic is $w_{1}=\frac{s}{s-1}$ and $w_{2}=\frac{t}{t-1}$.

Setting these conditions, we get

$$
l_{1}=\left|\ln \left(\frac{\alpha_{2}-\frac{2 t}{t-1}+1-\sqrt{1-\frac{4 t \alpha_{2}^{2}}{(t-1)}-2 \alpha_{2}^{2}+\frac{4 t^{2} \alpha_{2}^{4}}{(t-1)^{2}}-\frac{4 t \alpha_{2}^{4}}{t-1}+\alpha_{2}^{4}}}{\alpha_{2}-\frac{2 t \alpha_{2}^{2}}{t-1}+\alpha_{2}^{2}+\sqrt{1-\frac{4 t \alpha_{2}^{2}}{(t-1)}-2 \alpha_{2}^{2}+\frac{4 t^{2} \alpha_{2}^{4}}{(t-1)^{2}}-\frac{4 t \alpha_{2}^{4}}{t-1}+\alpha_{2}^{4}}}\right)\right|,
$$




$$
\begin{aligned}
& l_{2}=\left|\ln \left(\frac{\alpha_{2}^{2}-\frac{2 \alpha_{2}^{2} s}{s-1}+1+\sqrt{\alpha_{2}^{4}-\frac{4 \alpha_{2}^{4} s}{s-1}-2 \alpha_{2}^{2}+\frac{4 \alpha_{2}^{4} s^{2}}{(s-1)^{2}}-\frac{4 \alpha_{2}^{2} s}{s-1}+1}}{\alpha_{2}^{2}-\frac{2 \alpha_{2}^{2} s}{s-1}+1-\sqrt{\alpha_{2}^{4}-\frac{4 \alpha_{2}{ }^{4} s}{s-1}-2 \alpha_{2}^{2}+\frac{4 \alpha_{2} s^{2}}{(s-1)^{2}}-\frac{4 \alpha_{2}{ }^{2} s}{s-1}+1}}\right)\right|, \\
& l_{3}=\left|\ln \left(\frac{-s t \alpha_{2}^{2}+1+\alpha_{2}^{2}+\alpha_{2} \sqrt{s^{2} t^{2} \alpha_{2}^{2}-2 s t-2 s t \alpha_{2}^{2}+\alpha_{2}{ }^{2}-2+\alpha_{2}^{2}}}{-s t \alpha_{2}^{2}+1+\alpha_{2}^{2}-\alpha_{2} \sqrt{s^{2} t^{2} \alpha_{2}^{2}-2 s t-2 s t \alpha_{2}^{2}+\alpha_{2}{ }^{2}-2+\alpha_{2}^{2}}}\right)\right| .
\end{aligned}
$$

These represent the Fenchel-Nielsen coordinates using the coordinates $\alpha_{2}, s$ and $t$. It is clear that the condition for the length $l_{1}$ and $l_{2}$ to be the same can be reduced in this case to the condition $s=t$, which implies $s=t=w_{1}=w_{2}=u_{3}=v_{3}=2$.

Analogously to the way we evaluated the open pre-symplectic form on a trinion in the $\operatorname{PSL}(2, \mathbf{R})$ case, we are going to evaluate the open pre-symplectic form in the PGL $(3, \mathbf{R})$ case by explicitly evaluating the vector fields dual to the coordinates given. We then will be ready to integrate the symplectic form on the space of convex real projective structures. We will focus on the trinion case and in that case we will completely integrate the open pre-symplectic form defined on it. Since trinions represent the building blocks for Riemann surfaces of genus $g \geq 2$, utilising the glueings formula we proved we will be able to integrate the symplectic form for any closed Riemann surface.

The process is similar to the one already studied for the $\operatorname{PSL}(2, \mathbf{R})$ case, even though some technical difficulties are harder in the PGL(3,R) case.

Completely integrating the open pre-symplectic form for a "pair of pants" represents the first step towards the complete integration of the (open) symplectic form in its generality.

4.6. The symplectic form. In the previous chapter we discussed the coordinates for the space of convex real projective structures on $\Sigma(g, n)$. Given a trinion $P$ with boundary components $A, B, C$, satisfying the relation $C B A=1$, we will produce an explicit formula for the open pre-symplectic form in terms of the global coordinates that we defined in the previous chapter.

The first step is to describe the 1-cocycles in this particular case. Since 1-cocycles represent elements in the tangent space, it makes sense to try to describe them in terms of a basis. The dual of the set of coordinates represents a good basis for our vector space. In that way, the symplectic form would be completely described. Therefore we need to understand how to write the 1-cocycles corresponding to such a basis.

We will use the coordinates $l_{1}, l_{2}, l_{3}, m_{1}, m_{2}, m_{3}, s$ and $t$ that describe the space of structures on the trinion, in terms of the coordinates $\alpha_{1}, \alpha_{2}, \beta_{1}, \beta_{2}, w_{1}, w_{2}, s, t$ that geometrically define such structures. The next step is to evaluate the jacobian and its inverse-transpose $K=\left(J^{-1}\right)^{t}$.

Proceeding on the same line as in chapter 3 , we can now explicitly evaluate the vector fields, or the 1-cocycles, $\frac{\partial A}{\partial *}, \frac{\partial B}{\partial *}$, and $\frac{\partial C}{\partial *}$, where $*$ denotes a coordinate element.

We recall that the expression for the open pre-symplectic form on a trinion is

$$
\omega(\chi, \eta)=\operatorname{tr}\left(A^{-1} \chi(A) A \cdot \eta(B)+\chi(C) \cdot \eta(C)\right),
$$

where $\chi$ and $\eta$ are 1-cocycles. By substituting $\frac{\partial}{\partial *}$ for $\chi$ and $\eta$ we get the values of the entries for the open pre-symplectic form $\omega$.

By performing the relative calculations (for example using a computer program such as "Maple" [Red94]) substituting the relative entries, we then get the matrix 


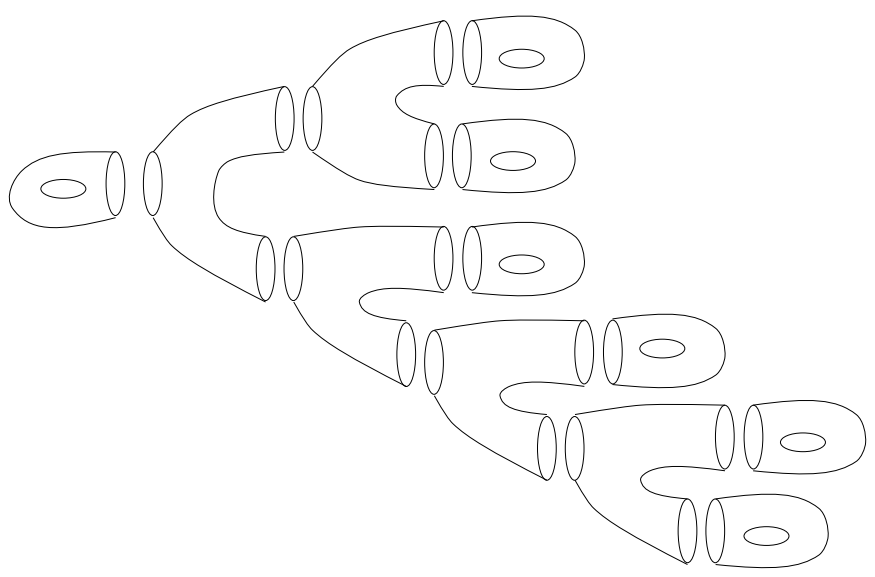

Figure 8. Tree of glued pants.

corresponding to the open pre-symplectic form, similarly to as we did for PSL $(2, \mathbf{R})$ (see $[$ Zoc95]).

4.7. Final remarks. In this final section we just wish to make a few comments on how to generalise the procedure explained in this article, and explicitly applied to the cases $G=\operatorname{PSL}(2, \mathbf{R})$ and $G=\operatorname{PGL}(3, \mathbf{R})$, to integrate the symplectic form on the moduli space of representations of the fundamental group of a compact Riemann surface into a reductive Lie group $G$.

The main item is clear: we need to be able to explicitly write the 1-cocycles corresponding to the tangent vectors on the space. Once we have their explicit expression we can evaluate the symplectic form on them.

In order to explicitly express the 1-cocycles corresponding to the tangent vectors, we needed to define coordinates $\xi_{j}$ on the space and, fixing a representation $\phi(\gamma)$, differentiate $\frac{\partial}{\partial \xi_{j}}(\phi)(\gamma)$ to produce a 1-cocyle that, to each element $\gamma$, associates an element in the Lie algebra $\mathcal{G}$ of $G$.

The glueing formulae, which have been proved in complete generality for any group $G$, enable us to simplify many calculations by focusing on a trinion.

In particular, we can glue the Riemann surface along one boundary component at a time. Our way of glueing the Riemann surface is represented by the tree of glued pants (see Figure 8).

\section{REFERENCES}

[AB82] M.F. Atiyah and R. Bott. The Yang-Mills-Equations over Riemann Surfaces. Philosophical Transactions of the Real Society of London, 308:523-615, 1982. MR 85k:14006

[AM95] A.Y. Alekseev and A.Z. Malkin. Symplectic Structure of the Moduli Space of Flat Connection on a Riemann Surface. Comm. Math. Phy. 169 (1995), 99-119. MR 96m: 58028

[Arm83] M.A. Armstrong. Basic Topology. Springer-Verlag, 1983. MR 84f:55001

[BK53] H. Busemann and P.J. Kelly. Projective Geometry and Projective Metrics. Academic Press, 1953. MR 14:1008e

[CG93] S. Choi and W.M. Goldman. Convex Real Projective Structures on Closed Surfaces are Closed. Proceedings of the American Mathematical Society, 118(2):657-661, 1993. MR 93g:57017 
[Fox53] R. Fox. The Free Differential Calculus. I: Derivations in the Group Ring. Annals of Mathematics, 57:547-560, 1953. MR 14:843d

[Gie80] D. Gieseker. Some Applications of Geometric Invariant Theory to Moduli Problems. Proc. Int. Congr. Math., Helsinki 1978, Vol. 2, 525-528, 1980. MR 81e:14007

[GM91] J. Gilman and B. Maskit. An Algorithm for 2-Generator Fuchsian Groups. Michigan Mathematical Journal, 38:13-31, 1991. MR 92f:30062

[GM88] W.M. Goldman and J.J. Millson. The Deformation Theory of Representations of Fundamental Groups of Compact Kaehler Manifolds. Publ. Math. I.H.E.S., 67:43-96, 1988. MR 90b:32041

[Gol84] W.M. Goldman. The Symplectic Nature of Fundamental Groups of Riemann Surfaces. Advances in Mathematics, 54:200-225, 1984. MR 86i:32042

[Gol86] W.M. Goldman. Invariant Functions on Lie Groups and Hamiltonian Flows of Surface Group Representations. Inventiones Mathematicae, 85:263-302, 1986. MR 87j:32069

[Go190] W.M. Goldman. Convex Real Projective Structures on a Riemann Surface. Journal of Differential Geometry, 31:791-845, 1990. MR 91b:57001

[Gol93] W.M. Goldman. Complex Hyperbolic Kleinian Groups. Complex Geometry (Osaka, 1990), Lecture Notes Pure Appl. Math., vol. 143, Marcel Dekker, New York, 1993, pp. 31-52. MR 93k:32053

[Gun66] R.C. Gunning. Lectures on Riemann Surfaces. Princeton University Press, 1966. MR 34:7789

[Har77] W.J. Harvey, editor. Discrete Groups and Automorphic Forms. Academic Press, 1977. MR 57:6413

[Hit92] N.J. Hitchin. Lie Groups and Teichmüller Space. Topology, 31(3):449-473, 1992. MR 93e: 32023

[Hop42] H. Hopf. Fundamentalgruppe und Zweite Bettische Gruppe. Commentari Mathematicae Helveticae, 14:257-309, 1942. MR 3:316e

[HJ94] J. Huebschmann and L.C. Jeffrey. Group Cohomology Construction of Symplectic Forms on Certain Moduli Spaces. Internat. Math. Res. Notices 1994, no. 6, $245 \mathrm{ff}$. (electronic) MR 95e:58033

[JM84] D. Johnson and J. Millson. Deformation Spaces of Compact Hyperbolic Manifolds. Discrete Groups in Geometry and Analysis, Proceedings of a Conference held at Yale University in honor of G.D. Mostow on his sixtieth birthday, March 1984, Birkhäuser, Boston, MA, 1987, pp. 48-106. MR 88j:22010

[Kar92] Y. Karshon. An Algebraic Proof for the Symplectic Structure of Moduli Space. Proceedings American Mathematical Society, 116:591-605, 1992. MR 93a:14010

[KM96] M. Kapovich and J.J. Millson. The Symplectic Geometry of Polygons in Euclidean Space. J. Differential Geom. 44 (1996), 479-513. CMP 97:07

[Kob82] S. Kobayashi. Invariant Distances for Projective Structures. Istituto Nazionale di Alta Matematica Symposium Mathematicae, 26:153-161, 1982. MR 83i:53037

[Kob84] S. Kobayashi. Projectively Invariant Distances for Affine and Projective Structures. Differential geometry, Banach Center Publications, 12:127-152, 1984. MR 89k:53043

[Lyn50] R.C. Lyndon. Cohomology Theory of Groups with a Single Defining Relation. Annals of Mathematics, 52(3):650-665, 1950. MR 13:819b

[MF82] D. Mumford and J. Fogarty. Geometric Invariant Theory. Springer-Verlag, 1982. MR 86a:14006

[Mil88] J.J. Millson. Deformations of Representations of Finitely Generated Groups. Contemporary Mathematics, 74:237-253, 1988. MR 90c:22034

[NS65] M.S. Narasimhan and C.S. Seshadri. Stable and Unitary Vector Bundles on a Compact Riemann Surface. Annals of Mathematics, 82:540-567, 1965. MR 32:1725

[Red94] D. Redfern. The Maple Handbook. Springer-Verlag, 1994.

[Thu] W. Thurston. The Geometry and Topology of 3-Manifolds. Lecture Notes.

[Wel80] R.O. Wells. Differential Analysis on Complex Manifolds. Springer-Verlag, 1980. MR 83f: 58001

[Wol82] S.A. Wolpert. The Fenchel-Nielsen Deformation. Annals of Mathematics, 115:501528, 1982. MR 83g:32024

[Wol83] S.A. Wolpert. On the Symplectic Geometry of Deformations of a Hyperbolic Surface. Annals of Mathematics, 117:207-234, 1983. MR 85e:32028 
[Wol85] S.A. Wolpert. On the Weil-Petersson Geometry of the Moduli Space of Curves. American Journal of Mathematics, 107:969-997, 1985. MR 87b:32040

[Wol88] S.A. Wolpert. Cut and Paste Deformations of Riemann Surfaces. Annales Academiae Scientiarum Fennicae, Ser. A I Math. 13:401-413, 1988. MR 90e:32026

[Wol89] S.A. Wolpert. Riemann Surfaces, Moduli and Hyperbolic Geometry. In M. Cornalba, X. Gomez-Mont, and A. Verjovsky, editors, Lectures on Riemann Surfaces, Trieste, Italy, 1987. International Centre of Theoretical Physics, World Scientific, 1989, pp. 48-98. MR 91i:32020

[Zoc95] V. Zocca. Integration of the Symplectic Form on the Space of Convex Real Projective Structures on a Riemann Surface. Doctoral Dissertation, University of Maryland, 1995.

Mathématiques, bÂt. 425, Université Paris XI, F-91405 Orsay Cedex, France

Current address: Department of Mathematics, University of Maryland, College Park, Maryland 20742

E-mail address: anz@math.umd.edu 